Theorem $X$. Ist die Spektralfunktion

$$
\chi^{0}(\varrho)=\lim _{n \rightarrow \infty} \chi_{n}{ }^{0}(\varrho)
$$

in jedem abgeschlossenen endlichen Intervall absolut stetig, so ist $\lim _{t \rightarrow \infty} R^{0}(t)=0$, und die betrachteten Systeme zeigen mit wachsendem $n$ für immer größere Zeitintervalle $0<t<T$ praktisch schwache Irreversibilität.

Wir können natürlich keinen expliziten Zusammenhang zwischen $n$ und $T$ geben, solange wir kein spezielles System vor Augen haben. Speziell für Kristalle mit harmonischer Wechselwirkung der Atome hat MAzUR ${ }^{8}$ kürzlich diesen Zusammenhang untersucht.

8 P. Mazur u. E. Montroll, J. Math. Phys. 1, 70 [1960].

\section{Schlußbemerkungen}

Man kann versuchen, der praktisch schwachen Irreversibilität eine praktisch starke Irreversibilität gegenüberzustellen. Doch liegen hierzu noch keine Ergebnisse vor. Worauf es uns hier ankam, war vor allem zu zeigen, daß die Irreversibilität in einfacher Weise mit gewissen Eigenschaften der Spektralfunktionen zusammenhängt.

Die Beweise der Theoreme und anderer Aussagen sind in den unter ${ }^{1}$ und ${ }^{2}$ zitierten Arbeiten enthalten. Der Beweis von Theorem VIII wird von KöNIG an anderer Stelle gegeben werden.

Herrn Kollegen H. KöNIG, Aachen, bin ich für sein Interesse an diesen Problemen und für seine tatkräftige und wertvolle Unterstützung in der Bereitstellung der mathematischen Grundlagen sehr zu Dank verpflichtet.

\title{
Zur Theorie der „seltsamen“ Teildhen
}

\author{
Von H.-P. DürR und W. Heisenberg \\ Aus dem Max-Planck-Institut für Physik und Astrophysik München \\ (Z. Naturforschg. 16 a, 726-747 [1961] ; eingegangen am 18. Mai 1961)
}

\begin{abstract}
The strange particles can be represented within the framework of the nonlinear spinor theory by taking into account the degeneracy with respect to isospin and parity of the groundstate "vacuum". Use is made of the mathematical analogy between the theory of superconductivity and the theory of elementary particles, and in a first approximation a fourfold degeneracy of the groundstate is assumed. Each auf the four states is considered as a mixture of states of similar symmetry. The GREen-functions of the type $\left\langle\Omega_{\alpha}\left|T X(x) \bar{X}\left(x^{\prime}\right)\right| \Omega_{\beta}\right\rangle$ are considered as invariant under the proper Lonentz-group, CPT and PG, applied on the field operators or the states $\left.\Omega_{\alpha}\right\rangle$ separately; but as invariant under isospin rotation, $\mathrm{P}$ or CT or G, only if the transformation is applied on the fieldoperators and the states $\left.\Omega_{a}\right\rangle$ simultaneously. Parity is represented in a manner discussed in an earlier paper by one of the authors. Only stationary states of strangeness 1 can be considered in this approximation. The fourfold degeneracy of the K-meson is reduced to an additional symmetry which may be connected later with the existence of electromagnetic charge. The results of the calculations may be interpreted by describing the strange particles as composed of ordinary particles and a "spurion" taken from the groundstate "vacuum". The "spurion" carries an isospin $1 / 2$ and a parity. The masseigenvalues and the parity of the particles are calculated by means of a slightly improved version of the $\mathrm{T}_{\mathrm{AMM}}-\mathrm{D}_{\mathrm{ANCOFF}}$-method. The theoretical masseigenvalues agree qualitatively with the observed masses. The calculated relative parities of the strange particles may later be checked by experiments. Besides the known particles of strangeness 1 , the theory yields other eigenstates which are probably highly unstable since they could disintegrate into more stable particles by means of strong or electromagnetic interactions.
\end{abstract}

In der Theorie der Elementarteilchen, die von der Gleichung

$$
\gamma_{\mu} \frac{\partial \psi}{\partial x_{\mu}} \pm l^{2} \gamma_{\mu} \gamma_{5} \psi\left(\bar{\psi} \gamma_{\mu} \gamma_{5} \psi\right)=0
$$

ausgeht, war zur Interpretation der "seltsamen“ Teilchen die Annahme vorgeschlagen worden ${ }^{1}$, da $\beta$ der Grundzustand "Welt“ entartet sei und einen sehr

1 H.-P. Dürr, W. Heisenberg, H. Mitter, S. Schlieder u. K. YaMAZAKI, Z. Naturforschg. 14 a, 441 [1959]; im folgenden als „A“ zitiert. hohen Isospin besitze; daß ferner ein Isospin 1/2 oder 1 von diesem Gesamtisospin abgezweigt und an ein Nukleon oder $\pi$-Meson angehängt werden könne. In dieser Weise sollten dann Hyperonen oder K-Mesonen entstehen. Im folgenden soll dieser Gedanke mathematisch durchgeführt werden. Dabei beschränken wir die Rechnungen auf die Teilchen von der „Seltsamkeit“ 1. Die Erweiterung auf Teilchen höherer Seltsamkeit wird nur am Schluß kurz gestreift, aber nicht mehr ausführlich behandelt werden. 


\section{Transformationseigenschaften und Vakuum- erwartungswerte}

\section{a) Zusammenhang mit dem Paritätsproblem}

Die empirische Tatsache, daß bei den schwachen Wechselwirkungen gleichzeitig die Erhaltung der „Seltsamkeit“ und die Erhaltung der Parität verlorengeht, legt die Vermutung nahe, daß eine enge Beziehung zwischen Seltsamkeit und Parität besteht. Es wird daher zweckmäßig sein, bei der Behandlung der seltsamen Teilchen von einer Darstellung der Gl. (1) auszugehen, in der die Parität unmittelbar in Erscheinung tritt.

Eine solche Darstellung ist von einem der Verfasser $^{2}$ angegeben worden. Es ist zunächst zweckmäßig, durch die Beziehungen

$$
\chi_{11}=\psi_{1}, \chi_{21}=\psi_{2}, \chi_{12}=-\psi_{4}{ }^{*}, \chi_{22}=\psi_{3}{ }^{*}
$$

einen neuen Feldoperator $\chi$ einzuführen, dessen erster Index den Dirac-Spin, dessen zweiter den Isospin bedeutet. Für diesen Operator $\chi$ lautet dann die Feldgleichung (1) :

$$
-i \sigma^{u} \frac{\partial \chi}{\partial x^{u}} \pm l^{2} \sigma^{\mu} \chi\left(\chi^{*} \sigma_{\mu} \chi\right)=0
$$

wobei $\sigma_{\mu}=\left(1, \sigma_{k}\right)$ und $\sigma^{\mu}=\left(-1, \sigma_{k}\right)$ ist und eine Einheitsmatrix im Isospinraum in der Schreibweise unterdrückt wurde. Die Raumspiegelung wird, um die Verbindung mit der sogen. „Parität 2. Art“ herzustellen, formal mit einem Vorzeichenwechsel des Parameters $l$ verknüpft durch eine Neudefinition der Koordinaten

$$
\xi_{k}=\frac{x_{k}}{l} ; \xi_{0}=\frac{x_{0}}{|l|} ; \pi_{k}=p_{k} l ; \pi_{0}=p_{0}|l| .
$$

Dies erlaubt die Einführung eines 8-komponentigen Spinoroperators $X(\xi,|l|)$ durch die Beziehung

$$
X\left(\vec{\xi}, \xi_{0},|l|\right)=\left(\begin{array}{l}
\chi\left(\vec{\xi}, \xi_{0},|l|\right. \\
\chi\left(\vec{\xi}, \xi_{0},-|l|\right.
\end{array}\right),
$$

der die üblichen Eigenschaften eines Dirac-IsospinOperators hat und für den insbesondere die Raumspiegelung $l \rightarrow-l$ oder $\chi\left(\vec{\xi}, \xi_{0}, l\right) \rightarrow \chi\left(-\vec{\xi}, \xi_{0},-l\right)$ wieder in der üblichen Form

$$
X\left(\vec{\xi}, \xi_{0},|l|\right) \rightarrow \Gamma_{4} X\left(-\vec{\xi}, \xi_{0},|l|\right)
$$

geschrieben werden kann. Wenn man nun zur Angleichung an frühere Bezeichnungsweisen eine neue

2 H.-P. DürR, Z. Naturforschg. 16 a, 327 [1961] ; im folgenden als „B“ zitiert.
Ortskoordinate $x=\xi|l|$ einführt, die nur für $l>0$ mit dem $x$ von (1) und (3) identisch ist, so lautet die Feldgleichung für diesen Operator :

$$
\begin{aligned}
\Gamma^{\mu} \frac{\partial}{\partial x^{\mu}} X \pm \frac{l^{2}}{2}\left[\Gamma_{5} \Gamma^{\mu} X(\right. & \left.\bar{X} \Gamma_{5} \Gamma_{\mu} X\right) \\
& \left.+\Gamma^{\mu} X\left(\bar{X} \Gamma_{\mu} X\right)\right]=0 .
\end{aligned}
$$

Ferner sollte für den Vakuumerwartungswert des Produktes aus zwei Feldoperatoren (ohne Berücksichtigung der seltsamen Teilchen) gelten:

$$
\begin{array}{r}
\left\langle 0\left|X(x) \bar{X}\left(x^{\prime}\right)\right| 0\right\rangle=(2 \pi)^{-4} \int \mathrm{d}\left(\varkappa^{2}\right) \int \mathrm{d}^{4} p e^{i p\left(x-x^{\prime}\right)} \\
\cdot\left[\frac{\Gamma_{\mu} p^{\mu}+i \varkappa}{p^{2}+\varkappa^{2}} \varrho_{1}\left(\varkappa^{2}\right)+\frac{\Gamma_{\mu} p^{\mu}-i \varkappa}{p^{2}+\varkappa^{2}} \varrho_{2}\left(\varkappa^{2}\right)\right],
\end{array}
$$

wobei nur $\varrho_{1}\left(\varkappa^{2}\right)$ einen zur Nukleonenmasse gehörigen $\delta$-Funktionsanteil haben soll. Die Annahme $\varrho_{1}\left(\varkappa^{2}\right) \neq \varrho_{2}\left(\varkappa^{2}\right)$ bedeutet, daß die Feldoperatoren $\chi(x,+|l|)$ und $\chi(x,-|l|)$ für zeitartige Abstände nicht antikommutieren. Da die Differentialgleichung die Funktionen $\chi(x,+|l|)$ und $\chi(x,-|l|)$ nicht miteinander verkoppelt, müßten sie aber antikommutieren, wenn sie zu irgendeinem Zeitpunkt als unabhängig betrachtet werden.

Die Nicht-Antikommutativität der Operatorfunktionen muß daher als Definition einer Abhängigkeit aufgefaßt werden. Man kann dann auch

$$
\chi(x,-|l|)=\tilde{\chi}(x)
$$

als Symbol für ein noch zu bestimmendes Funktional der Feldoperatoren $\chi(x,+|l|)=\chi(x)$ deuten:

$$
\tilde{\chi}(x)=F\left[\chi(x), \chi^{*}(x)\right] .
$$

Es soll durch die Forderung festgelegt werden können, $\operatorname{da} \beta \tilde{\chi}\left(-\vec{x}, x_{0}\right)$ sich bei allen zugelassenen Transformationen wie $\chi\left(\overrightarrow{\mathfrak{x}}, x_{0}\right)$ transformiert und einer Dgl. der Form (1) genügt und daß seine FouRIER-Transformierte auf der Massenschale des Nukleons mit der bezüglich der Vorschrift „Parität 2. Art“ umgeformten FourIER-Transformierten von $\chi(x)$ identisch ist. Wenn dies möglich ist, so lassen sich die Operatoren $\chi(x)$ und $\tilde{\chi}(x)$ formal zu einem Dirac-Isospin-Operator $X(x)$ vereinigen, der der Dgl. (7) gehorcht. Die Paritätssymmetrie der Theorie ist dann gewährleistet.

Dabei bleibt allerdings zunächst die Frage, ob eine solche Festlegung von $\tilde{\chi}(x)$ möglich ist. Legt man (7) und (8) einer Berechnung von Matrixelementen nach der „Neuen TAMm-DAncoff-Methode" zugrunde, so läßt sich in der Tat in jeder Näherung die funktionelle Abhängigkeit (9) in der Form von Beziehungen zwischen diesen Matrixele- 
menten bestimmen und damit wohl auch $\tilde{\chi}(x)$ wenigstens grundsätzlich berechnen und eliminieren. Die Frage nach der Möglichkeit der geschilderten Festlegung von $\tilde{\chi}(x)$ wird also auf immer höhere Näherungen, d. h. zu $\tau$-Funktionen von immer gröBerer Variabelnzahl verschoben. Die formale Einführung des 8-komponentigen Feldoperators (5) schiene damit schließlich nur zu bewirken, daß man in einer NTD-Rechnung eine Abschlußbedingung (Nullsetzen gewisser $\varphi$-Funktionen) für das unendliche, aus (3) folgende $\tau$-Gleichungssystem fordert, die gerade die Paritätssymmetrie in jeder Näherung gewährleistet. Allerdings ist einstweilen unklar, inwieweit man über diese Abschlußbedingung als eine Art „Randbedingung “ für die Theorie verfügen kann, ohne mit ihren Grundvoraussetzungen in Konflikt zu kommen. Diese Konvergenzprobleme der $\mathrm{T}_{\mathrm{AMM}}-\mathrm{DANCOFF}_{\mathrm{A}}$-Theorie sollen aber vorerst zurückgestellt werden; es soll also für das Folgende vorausgesetzt werden, daß die Randbedingungen für hohe Variabelnzahl die Paritätssymmetrie zulassen.

Diese hier kurz skizzierte Darstellung der Parität soll auch die Grundlage für die Rechnungen der vorliegenden Arbeit bilden.

\section{b) Analogie zu den Problemen von Supraleitung und Ferromagnetismus.}

$\mathrm{N}_{\mathrm{AMBU}}{ }^{3}$ hat kürzlich auf die enge mathematische Verwandtschaft hingewiesen, die zwischen der Theorie der Elementarteilchen und der BARDEEN-BogoLJUBowschen Form der Theorie der Supraleitung besteht. In beiden Fällen besitzt der Grundzustand offenbar nicht die volle Symmetrie der zugrunde gelegten Feldgleichung, sondern ist entartet. BogoLJUBow ${ }^{4}$ hat betont, daß es sich hier um einen gemeinsamen Zug einer ganzen Klasse von Problemen handelt, zu der neben der Theorie der Supraleitung auch die Theorie des Ferromagnetismus, der Kristallbildung, der Kondensation, der Suprafluidität usw. gehören. Probleme dieser Klasse können offenbar auf zwei verschiedene Weisen behandelt werden, die am Fall der Supraleitung kurz skizziert werden sollen:

Man kann entweder von dem entarteten Grundzustand $G\rangle$ ausgehen. Dann ist beim supraleitenden Metall die Zahl der Elektronen in diesem Zustand

3 Y. Nambu, Phys. Rev. Lett. 4, Nr. 7, 380 [1960] u. Bericht d. Rochester-Konferenz 1960, S. 858.

4 N. N. Bogolsubow, J. Exp. Theor. Phys., USSR 34 (7), 41 [1958] u. Vortrag auf d. Konferenz in Amsterdam 1959. nicht festgelegt. Der Erwartungswert

$$
\left\langle\boldsymbol{G}\left|\psi(x) \psi\left(x^{\prime}\right)\right| \boldsymbol{G}\right\rangle
$$

ist von Null verschieden - während er beim Normalleiter wegen der Eichgruppe verschwinden müßte und liefert den wichtigsten Beitrag zur sogenannten Energielücke; er bewirkt sozusagen die Supraleitung.

Oder man kann von einem nicht entarteten Grundzustand 0$\rangle$ ausgehen, der zu einer ganz bestimmten Elektronenzahl gehört. Man muß dann Vakuumerwartungswerte des Produktes von vier Operatoren vom Typus

$$
\left\langle 0\left|\psi(x) \psi\left(x^{\prime}\right) \psi^{*}(y) \psi^{*}\left(y^{\prime}\right)\right| 0\right\rangle
$$

betrachten ${ }^{5}$. Zwar muß jetzt wegen der Eichgruppe

$$
\left\langle 0\left|\psi(x) \psi\left(x^{\prime}\right)\right| 0\right\rangle=0
$$

gelten. Aber die Vakuumerwartungswerte vom Typus (11) brauchen auch dann nicht zu verschwinden, wenn der Abstand zwischen den Punktepaaren $x, x^{\prime}$ einerseits, $y, y^{\prime}$ andererseits sehr groß wird. Gewöhnlich pflegt man anzunehmen, daß im Grenzfall großen Abstandes zwischen den beiden Punktepaaren der Vakuumerwartungswert (11) in ein Produkt aus den Vakuumerwartungswerten

$$
\left\langle 0\left|\psi(x) \psi\left(x^{\prime}\right)\right| 0\right\rangle \text { und }\left\langle 0\left|\psi^{*}(y) \psi^{*}\left(y^{\prime}\right)\right| 0\right\rangle
$$

zerfallen müsse. Das ist hier nicht mehr richtig. Vielmehr wird jetzt der wichtigste Zwischenzustand einer sein, dessen Teilchenzahl um 2 höher ist als in $0\rangle$ - der Zustand soll hier einfach 2$\rangle$ genannt werden -, und im Grenzfall großen Abstandes gilt

$$
\begin{aligned}
& \left\langle 0\left|\psi(x) \psi\left(x^{\prime}\right) \psi^{*}(y) \psi^{*}\left(y^{\prime}\right)\right| 0\right\rangle \\
& \quad \approx\left\langle 0\left|\psi(x) \psi\left(x^{\prime}\right)\right| 2\right\rangle\left\langle 2\left|\psi^{*}(y) \psi^{*}\left(y^{\prime}\right)\right| 0\right\rangle .
\end{aligned}
$$

Die Matrixelemente $\left\langle 0\left|\psi(x) \psi\left(x^{\prime}\right)\right| 2\right\rangle$

spielen dann im wesentlichen die gleiche Rolle wie vorher der Ausdruck (10) und sind für die Entstehung der Supraleitung maßgebend.

Geht man nun von den Verhältnissen bei der Supraleitung wieder zur Theorie der Elementarteilchen über, so kann man im Sinne des zweiten eben geschilderten Verfahrens bei Annahme eines nicht entarteten Grundzustandes 4-Punktfunktionen vom Typus

$$
\left\langle 0\left|X(x) \bar{X}\left(x^{\prime}\right) X(y) \bar{X}\left(y^{\prime}\right)\right| 0\right\rangle
$$

5 Vgl. L. P. Gorkov, J. Exp. Theor. Phys., USSR 34 (7), 505 [1958]; ferner P. Mitrelstaedt, Z. Naturforschg., im Erscheinen. 
im Grenzfall großen Abstandes zwischen den beiden Punktepaaren $x, x^{\prime}$ und $y, y^{\prime}$ untersuchen. Soweit es sich um Beiträge von den Nukleonenzuständen handelt, wird (15) im wesentlichen in ein Produkt aus zwei Ausdrücken der Form (8) zerfallen. Man kann hier die Transformationen der Gruppe an den Faktoren $X(x) \bar{X}\left(x^{\prime}\right)$ bzw. $X(y) \bar{X}\left(y^{\prime}\right)$ getrennt vornehmen; die Invarianz besteht einzeln für jeden der beiden Faktoren. Es kann aber noch andere Beiträge geben, die bei Transformation nur eines Faktors eine geringere Symmetrie aufweisen. Insbesondere sollen Terme untersucht werden, die bei Transformation nur des einen Faktors zwar unter der eigentlichen LoRentz-Gruppe, CPT und $\mathrm{PG}^{6}$ invariant sind, die aber nicht notwendig unter Drehungen im Isospinraum und unter $P$ und $G$ einzeln in sich übergehen. Solche Terme können als Beiträge der Hyperonen gedeutet werden, wie nachher im einzelnen ausgeführt werden soll.

Da die Lonentz-, PG- und CPT-invarianten 2 Punktfunktionen die Gestalt haben

$$
\begin{aligned}
& F\left(x, x^{\prime}, \varkappa^{2}\right) \\
& =i\left[c_{1} \Gamma_{\mu} \frac{\partial}{\partial x_{\mu}}+c_{2} \Gamma_{\mu} \Gamma_{5} \frac{\partial}{\partial x_{\mu}}+c_{3} \varkappa+i c_{3+l} \varkappa \Gamma_{5} \tau_{l}\right] \\
& \cdot \Delta\left(x-x^{\prime}, \varkappa^{2}\right),
\end{aligned}
$$

wobei $c_{1}, \ldots, c_{6}$ reelle Konstanten und $\tau_{l}$ die Isospinmatrizen sind, so lautet die allgemeinste Form dieser weniger symmetrischen Beiträge der 4-Punktfunktion, die wir im Gegensatz zu den vollsymmetrischen Hauptgliedern als Zusatzglieder bezeichnen wollen, unter Weglassung der Integration über Massenspektren:

$$
\begin{gathered}
\left\langle 0\left|X_{\alpha \varkappa}(x) \bar{X}_{\beta \lambda}\left(x^{\prime}\right) X_{\gamma \mu}(y) \bar{X}_{\delta \nu}\left(y^{\prime}\right)\right| 0\right\rangle= \\
\ldots+\int \mathrm{d}^{4} p \mathrm{~d}^{4} q e^{i p\left(x-x^{\prime}\right)+i q\left(y-y^{\prime}\right)} \\
\cdot\left[A \frac{p_{\tau} \Gamma_{\alpha \varepsilon}^{\tau} \Gamma_{\varepsilon \beta}^{5}}{p^{2}+\varkappa_{1}^{2}} \cdot \frac{q_{\sigma} \Gamma_{\gamma \varphi}^{\sigma} \Gamma_{\varphi \delta}^{5}}{q^{2}+\varkappa_{2}^{2}} \delta_{\varkappa \lambda} \delta_{\mu \nu}\right. \\
\left.\quad+B \frac{\varkappa_{1} \varkappa_{2} \Gamma_{\alpha \beta}^{5} \Gamma_{\gamma \delta}^{5}\left(\tau_{\varkappa \lambda}^{l} \tau_{\mu \nu}^{l}\right)}{\left(p^{2}+\varkappa_{1}^{2}\right)\left(q^{2}+\varkappa_{2}^{2}\right)}\right]+\ldots .
\end{gathered}
$$

Man erkennt, daß der einzelne Faktor, z. B. $p_{\tau} \Gamma^{\tau} \Gamma^{5}$, bei der Transformation $\mathrm{P}$ oder $\mathrm{G}$ das Vorzeichen wechselt, daß aber der Gesamtausdruck bei allen Transformationen invariant ist. Der Term $\tau_{\varkappa \lambda}^{l} \tau_{\mu \nu}^{l}$, der die Ankopplung des Isospins ausdrückt, kann nur im Massenglied auftreten und ist dort zwangs-

$6 \mathrm{G}$ unterscheidet sich von $\mathrm{C}$ durch die Isospinumkehrung, vgl. T. D. LeE u. C. N. Y ANG, Nuovo Cim. 3, 749 [1956]. Die PG-Spiegelung der $\chi$-Operatoren ist in der üblichen läufig mit den Faktoren $\Gamma_{\alpha \beta}^{5} \Gamma_{\gamma \delta}^{5}$ verbunden, da nur so die PG-Invarianz des etwa von $X(y) \bar{X}\left(y^{\prime}\right)$ herrührenden Anteils für sich gewährleistet werden kann. Diese Verbindung mag auch der Grund dafür sein, daß bei den schwachen Wechselwirkungen die Erhaltung der $z$-Komponente des Isospins, der Seltsamkeit und der Parität gleichzeitig verlorengeht.

Nachdem in dieser Weise die Symmetrieabweichung der Zweipunktfunktionen festgestellt ist, kann man in Analogie zu den Verhältnissen bei der Supraleitung wieder zur ersten Darstellung mit einem entarteten Grundzustand übergehen. Solange man sich auf Beiträge der Teilchen von der Seltsamkeit 1 beschränkt, wird es genügen, einen vierfachen Grundzustand zu betrachten, dessen Komponenten durch die beiden Isospinwerte $1 / 2$ und $-1 / 2$ und eine rechts-links-Symmetrie (Helizität) charakterisiert werden.

\section{c) Der Raum der vier Grundzustände}

Im Raum der vier Grundzustände wollen wir als Operatoren die PAuli-Matrizen $\Sigma_{k}$ und $\varrho_{k}$ einführen. Die Paritätsoperation soll durch $\Sigma_{1}$, die Drehung im Isospinraum durch $e^{i \frac{1}{2} a_{l} \varrho_{l}}$ dargestellt werden.

Die Zustände sollen, ebenso wie es bei dem symmetrischen Grundzustand 0$\rangle$ möglich wäre, auch als Gemenge aufgefaßt werden können. Die verschiedenen Zustände des Gemenges sollen sich aber in bezug auf Parität und Isospindrehung gleich verhalten.

Bezeichnet man den Zustand, gleichgültig, ob es sich um einen reinen Fall oder ein Gemenge handelt, mit $\left.\Omega_{a}\right\rangle$, wobei der Index $\alpha$ der vier Werte fähig ist, so wäre also das Matrixelement eines Operators $O$ im Raum dieser Zustände mit

$$
\left\langle\Omega_{\alpha}|O| \Omega_{\beta}\right\rangle=O_{a \beta}
$$

zu bezeichnen, wobei man im Falle eines Gemenges die linke Seite als

$$
\left\langle\Omega_{a}|O| \Omega_{\beta}\right\rangle=\sum g_{n}\left\langle\Omega_{a}{ }^{n}|O| \Omega_{\beta}{ }^{n}\right\rangle
$$

aufzufassen hätte. $\left.\Omega_{a}{ }^{n}\right\rangle$ sind die Komponenten des Gemenges, $g_{n}$ die zugehörigen Gewichte. Es wird sich später als notwendig erweisen, die Zustände $\left.\Omega_{\alpha}\right\rangle$ als Gemenge zu interpretieren, wenn man genau die Symmetrieeigenschaften darstellen will, die hier gefordert werden.

Darstellung durch $\quad \chi(\mathrm{r}, t) \rightarrow\left(i \tau_{\mathbf{2}}\right)\left(i \sigma_{\mathbf{2}}\right) \chi^{* T}(-\mathrm{r}, t) \quad$ gegeben. 
Neben den schon besprochenen Transformationen Parität und Isospindrehung spielen noch die weiteren Transformationen GP und CPT eine wichtige Rolle. Wenn man ferner die Umklappung des Isospins ${ }^{6}$ mit $\mathrm{J}$ bezeichnet, also

$$
\mathrm{G}=\mathrm{C} \cdot \mathrm{J}
$$

setzt und damit $\mathrm{CPT}=\mathrm{GP} \cdot \mathrm{JT}$ erhält, so kann man die Darstellung dieser Operationen durch folgende Formeln definieren $(O$ sei zunächst ein Operator, der nur im Raum der $\left.\Omega_{a}\right\rangle$ wirkt) :

$\left\langle\mathrm{CPT} \Omega_{\alpha}|O| \operatorname{CPT} \Omega_{\beta}\right\rangle=O_{\beta \alpha}^{*}$,

$\left\langle\mathrm{GP} \Omega_{\alpha}|O| \mathrm{GP} \Omega_{\beta}\right\rangle=\left(i \varrho_{2} \Sigma_{1}\right)_{\alpha \gamma} O_{\delta \gamma}\left(-i \varrho_{2} \Sigma_{1}\right)_{\delta \beta}$,

$\left\langle\mathrm{JT} \Omega_{\alpha}|O| \mathrm{JT} \Omega_{\beta}\right\rangle=\left(i \varrho_{2} \Sigma_{1}\right)_{\alpha \gamma} O_{\gamma \delta}^{*}\left(-i \varrho_{2} \Sigma_{1}\right)_{\delta \beta}$.

[" bedeutet Komplexkonjugation; $i \varrho_{2}$ ist in der normalen PAULI-Darstellung der „Umkehr“operator : $\left.\varrho_{l}^{\mathrm{T}}=-\left(i \varrho_{2}\right) \varrho_{l}\left(-i \varrho_{2}\right)\right]$. Die Darstellung (21) bis (23) ist der Darstellung der gleichen Transformationen in den Matrizen $\Gamma_{\mu}$ und $\tau_{l}$ in (17) nachgebildet. Es entspricht etwa $\varrho_{k} \operatorname{dem} \tau_{k}, \Sigma_{1} \operatorname{dem} \Gamma_{4}$, $\Sigma_{2}$ der „Helizität“ $\Gamma_{5}$. Wenn der Operator $O$ auch in anderen Räumen wirksam ist, so muß noch definiert werden, was die Operation „Stern * “ bedeuten soll. Wir setzen etwa

$$
O=\sum_{i} O_{i} a_{i},
$$

wobei die $O_{i}$ nur im Raum der $\left.\Omega_{a}\right\rangle$ wirken sollen (d.h. durch die $\varrho_{k}$ und $\Sigma_{k}$ und $c$-Zahlen ausgedrückt werden können), während die $a_{v}$. Operatoren nur in anderen Räumen sein können. Dann soll $a_{i}^{*}$ das hermitesch konjugierte zu $a_{i}$ bedeuten ${ }^{7}$. Damit sind die notwendigen Transformationen in ihrer Darstellung festgelegt.

In I b) war gefordert worden, daß die Grundzustände $\left.\Omega_{a}\right\rangle$, von denen die Theorie ausgehen soll, gegenüber den Transformationen GP und CPT invariant sind. Man erreicht dies am einfachsten, indem man einen Zustand $\left.\Omega_{a}\right\rangle$, bei dem dies zunächst vielleicht nicht der Fall ist, in ein Gemenge aus den Zuständen $\left.\Omega_{a}\right\rangle$, GP $\left.\Omega_{a}\right\rangle$; CPT $\left.\Omega_{a}\right\rangle$ und JT $\left.\Omega_{a}\right\rangle$ mit gleichen Gewichten überführt. Dabei geht der Ope-

7 Betrachtet man die Vakuum-2-Punktfunktionen, so treten Ausdrücke der Form (16) auf, wobei nun die Konstanten $c_{i}$ von den Vakuumzuständen derart abhängen, daß der Vakuumerwartungswert insgesamt bei allen zugelassenen Transformationen der Theorie invariant bleibt. Insbeson- rator $\boldsymbol{O}_{\alpha \beta}$ in den „symmetrisierten“ Operator

$O_{\alpha \beta} \rightarrow \frac{1}{4}\left[O_{\alpha \beta}+O_{\beta \alpha}^{*}\right.$

$$
\left.+\left(i \varrho_{2} \Sigma_{1}\right)_{\alpha \gamma}\left(O_{\delta \gamma}+O_{\gamma \delta}^{*}\right)\left(-i \varrho_{2} \Sigma_{1}\right)_{\delta \beta}\right]
$$

über. Bei diesem Symmetrisierungsprozeß bleiben von den Grundoperatoren 1, $\varrho_{k}, \Sigma_{k}$ nur die folgenden übrig:

$$
1, \Sigma_{1}, \Sigma_{2}, \Sigma_{3} \varrho_{k} \text {. }
$$

Jeder lineare Ausdruck aus diesen Operatoren mit reellen (oder im Falle von Operatoren in anderen Räumen: hermiteschen) Koeffizienten ist im Raume der GP- und CPT-symmetrischen Grundzustände als Vakuumerwartungswert möglich.

\section{d) Darstellung der Erwartungswerte}

Durch die Einführung der Operatoren (26) lassen sich die Glieder (17) der 4-Punktfunktion in den Zusatzgliedern der Vakuumerwartungswerte von zwei Feldoperatoren nachbilden. Diese lauten unter Weglassung der Integration über das Massenspektrum [vgl. Gl. (8) ] :

$$
\begin{aligned}
& \left\langle\Omega\left|X(x) \bar{X}\left(x^{\prime}\right)\right| \Omega\right\rangle=\ldots+\int \mathrm{d}^{4} p e^{i p\left(x-x^{\prime}\right)} \\
& \quad \cdot\left[A \frac{p_{\nu} \Gamma^{v} \Gamma_{5} \Sigma_{2}}{p^{2}+\varkappa^{2}}+B \frac{i \varkappa}{p^{2}+\varkappa^{2}} i \Gamma_{5} \Sigma_{3}\left(\varrho^{l} \tau^{l}\right)\right] .
\end{aligned}
$$

Da zu den im Raum der $\left.\Omega_{a}\right\rangle$ zugelassenen Operatoren nach (26) auch $\Sigma_{1}$ gehört, können die seltsamen Teilchen zu den [in (27) nicht angegebenen] Hauptgliedern dieser Funktionen grundsätzlich auch Glieder beitragen, die den Operator $\Sigma_{1}$ (der ja die volle Symmetrie bei allen diskutierten Transformationen besitzt) enthalten. Der Operator $\Sigma_{1}$ kann also zwar nicht bei den (bezüglich der Vakuumtransformationen unsymmetrischen) Zusatzgliedern (27), wohl aber in den (symmetrischen) Hauptgliedern auftreten. Es wird sich jedoch später herausstellen, daß die Forderung der Konsistenz zwischen Eigenwertgleichung und 2-Punktfunktion die Annahme zuläßt, daß der Beitrag dieser Glieder sehr klein ist und vorläufig in hinreichender Näherung vernachlässigt werden kann.

In der Tamm-Dancoff-Methode werden die 2Punktfunktionen zur Abschätzung von Matrixelementen für zeitgeordnete Produkte von mehr als zwei Feldoperatoren benützt, indem das Verfahren der Kontraktion entsprechend der W $\mathrm{W}_{\text {IcKschen Regel an- }}$

dere entspricht der Forderung der CPT-Invarianz und JTInvarianz die Hermitezitätsforderung für den „Operatoranteil“", d. h. für die mit $\Gamma_{4}$ multiplizierte rechte Seite von (16) . 
gewandt wird. Es muß nun noch untersucht werden, wie das Kontraktionsverfahren auszuführen ist, wenn eine Entartung des Grundzustandes wie in (27) vorliegt. Zum Beispiel kommen bei der Näherungsdarstellung von

$$
\left\langle\Phi\left|\mathrm{T} X(x) \bar{X}\left(x^{\prime}\right) X(y) \bar{X}\left(y^{\prime}\right) X(z)\right| \Omega\right\rangle
$$

Ausdrücke wie

$$
\begin{aligned}
&\langle\Phi|X(x)| \Omega\rangle\left\langle\Omega\left|\mathrm{T} X(z) \bar{X}\left(x^{\prime}\right)\right| \Omega\right\rangle \\
&\left\langle\Omega\left|\mathrm{T} X(y) \bar{X}\left(y^{\prime}\right)\right| \Omega\right\rangle
\end{aligned}
$$

oder der bei nichtentartetem Vakuum gleiche Ausdruck

$$
\begin{array}{r}
\langle\Phi|X(x)| \Omega\rangle\left\langle\Omega\left|\mathrm{T} X(y) \bar{X}\left(y^{\prime}\right)\right| \Omega\right\rangle \\
\left\langle\Omega\left|\mathrm{T} X(z) \bar{X}\left(x^{\prime}\right)\right| \Omega\right\rangle
\end{array}
$$

vor. Bei entartetem Vakuum hat es zunächst den Anschein, als ob die beiden Ausdrücke wegen der Nichtvertauschbarkeit der $\Sigma_{k^{-}}$oder $\varrho_{k}$-Matrizen nicht gleich wären. Da die Grundzustände aber als Gemenge aus $\left.\Omega_{a}\right\rangle$ und CPT $\left.\Omega_{a}\right\rangle$ gebildet und damit CPT-invariant sind, wird bei der Symmetrisierung (25) von selbst auch die Reihenfolge der Faktoren symmetrisiert, d. h. alle Produkte im Raum der $\varrho_{k}$, $\Sigma_{k}$ sind von vornherein als symmetrisierte Produkte $O_{i} \cdot O_{k}=\frac{1}{2}\left(O_{i} O_{k}+O_{k} O_{i}\right)$ definiert. Bei den Rechnungen der folgenden Abschnitte wird daher auch immer von dieser symmetrisierten Produktbildung Gebrauch gemacht werden.

Es soll nun noch im einzelnen gezeigt werden, $\mathrm{da} ß$ eine Eigenwertgleichung für die Matrixelemente, die den oben angegebenen Symmetrieforderungen genügt, auch zu einem Erwartungswert mit den Symmetrien der Form (27) führt. Zunächst eine allgemeine Bemerkung über die Symmetrie (25). Sei $O$ ein Operator der Symmetrie (25), der nicht nur im Raum der Zustände $\left.\Omega_{a}\right\rangle$ wirkt, und $\varphi$ ein Matrixelement, das der Eigenwertgleichung

$$
O_{a \beta} \varphi_{\beta}=0
$$

genügt, so kann man durch

$$
\chi_{\gamma}^{*}=\left(i \varrho_{2} \Sigma_{1}\right)_{\gamma \delta} \varphi_{\delta}
$$

ein zweites Matrixelement konstruieren, das der Gleichung

$$
O_{\alpha \beta} \chi_{\alpha}^{*}=0
$$

genügt. $\chi_{a}{ }^{*}$ gehört also zum gleichen Eigenwert wie $\varphi_{a}$ und unterscheidet sich von $\varphi_{a}$ nur dadurch, daß sich $\varphi_{a}$ auf die „ket"-Seite, $\chi_{a}^{*}$ auf die „bra“-Seite der Vektoren der Grundzustände bezieht. Bildet man aus $\varphi_{a}$ und $\chi_{a}{ }^{*}$ die zu diesem Eigenwert gehörigen Beiträge zur 2-Punktfunktion, so erhält man wegen der geforderten Symmetrie in „bra“ und „,ket“ für diese Funktion

$$
\varphi_{a}^{*} \varphi_{\beta}+\chi_{\alpha}^{*} \chi_{\beta} .
$$

Man erkennt sofort, daß dieser Ausdruck (34) wieder die Symmetrie (25) besitzt. Die Gln. (31) und (33) können auch so interpretiert werden, daß $\varphi_{a}$ jene Matrixelemente bedeutet, die vom Eigenzustand zu irgendeinem "ket"-Vektor des Gemenges $\Omega\rangle$ führen, während die $\chi_{\alpha}$ zu einem „bra“-Vektor des Gemenges leiten.

Nach diesen Vorbemerkungen betrachten wir eine Eigenwertgleichung für $\varphi$ der geforderten Symmetrie:

$$
\begin{aligned}
& \left\{\Gamma^{\mu} p_{\mu}\left(1+\alpha \Sigma_{2} \Gamma_{5}\right)\right. \\
& \left.-i \varkappa\left[\beta+i \gamma \Sigma_{3} \Gamma_{5}(\varrho \tau)\right]\right\} \varphi=0
\end{aligned}
$$

oder im Ruhsystem des Teilchens

$\left\{p_{0}\left(1+\alpha \Sigma_{2} \Gamma_{5}\right)\right.$

$$
\left.-\varkappa\left[\beta \Gamma_{4}+\gamma \Sigma_{3} i \Gamma_{4} \Gamma_{5}(\varrho \tau)\right]\right\} \varphi=0 .
$$

Die beiden Operatoren $(\varrho \tau)$ und $\Sigma_{1} \Gamma_{4}$ sind mit dem Klammerausdruck \{\} vertauschbar und können gleichzeitig auf Diagonalform gebracht werden. Setzt man

$$
\Sigma_{1} \Gamma_{4} \cdot(\varrho \tau)=\varepsilon,
$$

so hat $\varepsilon$ die Eigenwerte \pm 1 und \pm 3 , und an die Stelle von (36) tritt:

$$
\left\{p_{0}\left(1+\alpha \Sigma_{2} \Gamma_{5}\right)-\varkappa\left(\beta \Gamma_{4}+\gamma \varepsilon \Sigma_{2} \Gamma_{5}\right)\right\} \varphi=0 .
$$

Durch Multiplikation mit

$$
\left\{p_{0}\left(-1+\alpha \Sigma_{2} \Gamma_{5}\right)-\varkappa\left(\beta \Gamma_{4}+\gamma \varepsilon \Sigma_{2} \Gamma_{5}\right)\right\}
$$

ergibt sich als Bestimmungsgleichung für $p_{0}$ :

$$
p_{0}^{2}-\left(\alpha p_{0}-\gamma \varepsilon \varkappa\right)^{2}-\varkappa^{2} \beta^{2}=0
$$

oder

$p_{0}=-\frac{a \gamma \varepsilon}{1-a^{2}} \varkappa \pm \varkappa \frac{\sqrt{\beta^{2}-\alpha^{2} \beta^{2}+\gamma^{2} \varepsilon^{2}}}{1-\alpha^{2}}=-a \varepsilon \varkappa \pm \varkappa b\left(\varepsilon^{2}\right)$.

Wenn man die Summation über die aus dem ruhenden Teilchen durch LoREnTz-Transformation hervorgehenden Zustände bis an den Schluß verschiebt, so lautet der von diesen beiden Zuständen herrührende Beitrag zur Summe über $\varphi^{*} \varphi$ wie in der üblichen Theorie

$$
\frac{p_{0}\left(-1+\alpha \Sigma_{2} \Gamma_{5}\right)-\varkappa\left(\beta \Gamma_{4}+\gamma \varepsilon \Sigma_{2} \Gamma_{5}\right)}{p_{0}{ }^{2}-\left(\alpha p_{0}-\gamma \varepsilon \varkappa\right)^{2}-\varkappa^{2} \beta^{2}} .
$$

Die Zustände zu $\varepsilon=-1,-3$ sind die Antiteilchen der Zustände zu $\varepsilon=+1,+3$. Faßt man die Beiträge zu 
$\varepsilon=+1$ und $\varepsilon=-1$ zusammen, so ergibt sich

$$
\begin{aligned}
\frac{9-\varepsilon^{2}}{8}\left\{\frac{1+\varepsilon}{2} \frac{p_{0}\left(-1+\alpha \Sigma_{2} \Gamma_{5}\right)-\varkappa\left(\beta \Gamma_{4}+\gamma \Sigma_{2} \Gamma_{5}\right)}{p_{0}^{2}-\left(\alpha p_{0}-\gamma \varkappa\right)^{2}-\varkappa^{2} \beta^{2}}+\frac{1-\varepsilon}{2} \frac{p_{0}\left(-1+\alpha \Sigma_{2} \Gamma_{5}\right)-\varkappa\left(\beta \Gamma_{4}-\gamma \Sigma_{2} \Gamma_{5}\right)}{p_{0}^{2}-\left(\alpha p_{0}-\gamma \varkappa\right)^{2}-\varkappa^{2} \beta^{2}}\right\} \\
=\frac{9-\varepsilon^{2}}{8} \cdot \frac{A+\varepsilon \gamma B}{\left[p_{0}^{2}-\left(\alpha p_{0}-\gamma \varkappa\right)^{2}-\varkappa^{2} \beta^{2}\right]\left[p_{0}^{2}-\left(\alpha p_{0}+\gamma \varkappa\right)^{2}-\varkappa^{2} \beta^{2}\right]} . \\
A=-\left[p_{0}+\varkappa \beta \Gamma_{4}\right]\left[p_{0}^{2}\left(1-\alpha^{2}\right)-\varkappa^{2}\left(\beta^{2}+\gamma^{2}\right)\right]+\alpha p_{0} \Sigma_{2} \Gamma_{5}\left[p_{0}^{2}\left(1-\alpha^{2}\right)-\varkappa^{2}\left(\beta^{2}-\gamma^{2}\right)\right], \\
B=\varkappa\left\{2 \alpha p_{0}\left[p_{0}+\varkappa \beta \Gamma_{4}\right]-\Sigma_{2} \Gamma_{5}\left[p_{0}{ }^{2}\left(1+\alpha^{2}\right)-\varkappa^{2}\left(\beta^{2}+\gamma^{2}\right)\right]\right\} .
\end{aligned}
$$

Die Beiträge zu $\varepsilon= \pm 3$ erhält man aus $(42-44)$, indem man den Projektionsoperator $\left(9-\varepsilon^{2}\right) / 8$ durch $\left(\varepsilon^{2}-1\right) / 8$ und in $A$ und $B$ und im Nenner $N$ die Größe $\gamma^{2}$ durch $9 \gamma^{2}$ ersetzt. Benützt man noch (37), so ergibt sich als Summe

$$
\frac{3+(\varrho \tau)}{4} \cdot \frac{A(1)+\Sigma_{1} \Gamma_{4} \gamma B(1)}{N(1)}+\frac{1-(\varrho \tau)}{4} \cdot \frac{A(9)-3 \Sigma_{1} \Gamma_{4} \gamma B(9)}{N(9)} .
$$

Fügt man nun noch den Ausdruck $\chi^{*} \chi$ entsprechend (34) hinzu, so folgt schließlich:

$$
\begin{aligned}
\frac{1}{2}\left[\frac{3 A(1)}{N(1)}\right. & \left.+\frac{A(9)}{N(9)}\right]-\frac{i \Gamma_{4} \Gamma_{5} \Sigma_{3}(\varrho \tau)}{2} \gamma \varkappa\left[\frac{p_{0}^{2}\left(1+\alpha^{2}\right)-\varkappa^{2}\left(\gamma^{2}+\beta^{2}\right)}{N(1)}+3 \frac{p_{0}{ }^{2}\left(1+\alpha^{2}\right)-\varkappa^{2}\left(9 \beta^{2}+\gamma^{2}\right)}{N(9)}\right] \\
& +3 \Gamma_{4} \Sigma_{1} \gamma \varkappa \alpha p_{0}\left(p_{0}+\varkappa \beta \Gamma_{4}\right)\left(\frac{1}{N(1)}-\frac{1}{N(9)}\right) .
\end{aligned}
$$

Geht man durch eine LoRENTz-Transformation zu einembewegten System über, so erhält man für diesen Ausdruck, wenn man noch mit $i \Gamma_{4}$ von rechts multipliziert:

$$
K_{1}\left(p^{2}\right)\left(\Gamma_{\mu} p^{u}+i \beta \varkappa\right)+K_{2}\left(p^{2}\right) p^{\mu} \Gamma_{\mu} \Gamma_{5} \Sigma_{2}+K_{3}\left(p^{2}\right)(i \varkappa) \cdot i \Gamma_{5} \Sigma_{3}(\varrho \tau)+K_{4}\left(p^{2}\right) \Sigma_{1}\left(\Gamma_{\mu} p^{\mu}+i \frac{\left(-p^{2}\right)}{\beta \varkappa}\right),
$$

wobei die $K_{i}\left(p^{2}\right)$ gegeben sind durch

$$
\begin{gathered}
K_{1}\left(p^{2}\right)=\frac{1}{2}\left[3 N^{\prime}(1)^{-1}\left(p^{2}\left(1-\alpha^{2}\right)+\varkappa^{2}\left(\beta^{2}+\gamma^{2}\right)\right)+N^{\prime}(9)^{-1}\left(p^{2}\left(1-\alpha^{2}\right)+\varkappa^{2}\left(\beta^{2}+9 \gamma^{2}\right)\right)\right], \\
K_{2}\left(p^{2}\right)=-\frac{\alpha}{2}\left[3 N^{\prime}(1)^{-1}\left(p^{2}\left(1-\alpha^{2}\right)+\varkappa^{2}\left(\beta^{2}-\gamma^{2}\right)\right)+N^{\prime}(9)^{-1}\left(p^{2}\left(1-\alpha^{2}\right)+\varkappa^{2}\left(\beta^{2}-9 \gamma^{2}\right)\right)\right], \\
K_{3}\left(p^{2}\right)=\frac{\gamma}{2}\left[N^{\prime}(1)^{-1}\left(p^{2}\left(1+\alpha^{2}\right)+\varkappa^{2}\left(\beta^{2}+\gamma^{2}\right)\right)+3 N^{\prime}(9)^{-1}\left(p^{2}\left(1+\alpha^{2}\right)+\varkappa^{2}\left(\beta^{2}+9 \gamma^{2}\right)\right)\right], \\
K_{4}\left(p^{2}\right)=3 \alpha \beta \gamma \varkappa^{2}\left[N^{\prime}(1)^{-1}-N^{\prime}(9)^{-1}\right] \\
\text { und die Nenner } \quad N^{\prime}(1)=\left(1-\alpha^{2}\right)^{2}\left[p^{2}+(b(1)+a)^{2} \varkappa^{2}\right]\left[p^{2}+(b(1)-a)^{2} \varkappa^{2}\right], \\
N^{\prime}(9)=\left(1-\alpha^{2}\right)^{2}\left[p^{2}+(b(9)+a)^{2} \varkappa^{2}\right]\left[p^{2}+(b(9)-a)^{2} \varkappa^{2}\right],
\end{gathered}
$$

und $a, b\left(\varepsilon^{2}\right)$ von Gl. (40).

Man erkennt, daß der Ausdruck (47) nur wieder Glieder der geforderten Symmetrie hat. Insbesondere kommt der für die Unterscheidung zwischen Singletts und Tripletts maßgebende Operator $(\varrho \tau)$ nur in der Verbindung $i \Gamma_{5} \Sigma_{3}(\varrho \tau)$ vor. Da sich später herausstellen wird, da $\gamma \ll 1$ ist, und da $N^{\prime}(9)$ sich von $N^{\prime}(1)$ nur um Glieder der Ordnung $\gamma^{2}$ unterscheidet, wird das letzte Glied in (47) sehr klein $\left(\sim \gamma^{3}\right)$; es soll daher, wie schon oben erwähnt, vernachlässigt werden. Der Ausdruck (47) entspricht dann im wesentlichen wieder dem Ansatz (27).

Aus Gl. (40) geht hervor, daß es als Lösung der Eigenwertgleichung zwei Singlett- und zwei Triplettzustände und die zugehörigen Antiteilchen gibt. Die vier Massenwerte sind im allgemeinen verschieden. Die beiden Singlett-Teilchen unterscheiden sich im Ruhsystem durch das Vorzeichen von $\Sigma_{1} \Gamma_{4}$, also der Parität; ebenso die beiden Triplett-Teilchen. Wenn die Massendifferenzen hinreichend hoch sind, werden die
Teilchen mit größerer Masse wohl durch Emission von $\pi$-Mesonen mit starker Wechselwirkung in solche mit niedrigerer Masse übergehen können, also instabil sein.

\section{Abschätzung der Masseneigenwerte}

a) Erweiterung der TAMм-Dancoff-Methode

Die Tamm-Dancoff-Methode geht von der Vorschrift aus, daß man neben den Matrixelementen zeitgeordneter Operatorprodukte, den sog. $\tau$-Funktionen, einen zweiten Satz von Funktionen, die sog. $\varphi$-Funktionen, durch den Prozeß der Kontraktion konstruieren solle. Für die $\varphi$-Funktionen wird dann die Annahme gemacht, daß sie von einer gewissen Variabelnzahl ab als sehr klein vernachlässigt werden können und daß in dieser Weise ein mit wach- 
sender Variabelnzahl konvergentes Rechenverfahren entstehe. Man konstruiert also aus einer $\tau$-Funktion durch Anwendung der Feldgleichung (1), (3) oder (7) eine $\tau$-Funktion mit einer um 2 höheren Variabelnzahl und führt diese Funktion durch Kontraktion unter Vernachlässigung der $\varphi$-Funktion der höheren Variabelnzahl auf eine $\tau$-Funktion kleinerer Variabelnzahl zurück. Da man die Dgl. auf jede der Variabeln einzeln anwenden kann, ist die Zahl der zur Bestimmung der $\tau$-Funktionen dienenden Eigenwertgleichungen, die in dieser Weise gebildet werden können, im allgemeinen höher als die Zahl der unbekannten $\tau$-Funktionen.

Die dadurch herbeigeführte Überbestimmung ist bisher meist in der Weise vermieden worden, daß man aus mehreren verschiedenen Integralkernen zur gleichen $\tau$-Funktion geeignete Mittelwerte gebildet hat, so daß schließlich nur eine einzige, gemittelte Eigenwertgleichung übrig blieb ${ }^{8}$. Da dabei aber die Gefahr besteht, daß die einzelne Integralgleichung auch nicht mehr näherungsweise erfült ist, soll hier ein etwas anderes Verfahren eingeschlagen werden. Wir wollen fordern, daß alle Integralgleichungen gleichzeitig näherungsweise, d. h. etwa mit dem gleichen mittleren Fehler erfüllt seien. Die höheren $\varphi$ Funktionen geben dabei ein $\mathrm{Ma}$ für den mittleren Fehler; wir fordern also, daß die integrierte Quadratsumme der höheren $\varphi$-Funktionen ein Minimum sein solle ${ }^{9}$. Der Eigenwert wird dann durch die Forderung bestimmt, daß diese Quadratsumme für den betreffenden Energiewert am kleinsten sein muß.

Schematisch läßt sich das mathematische Verfahren etwa in folgender Weise darstellen (wir ersetzen dabei die Integralgleichungen durch gewöhnliche lineare Gleichungen). Gegeben sei eine Anzahl linearer Gleichungen

$$
\sum_{k=1}^{n} a_{\lambda k}(E) \tau_{k}=\xi_{\lambda} \quad(\lambda=1, \ldots, N) .
$$

mit $N>n$. Die $\xi_{\lambda}$ sind dabei als entstanden zu denken durch Anwendung irgendwelcher Integraloperationen auf die höheren $\varphi$-Funktionen. Im Normalfall wäre $N=n$ und es würde $\xi_{\lambda}=0$ gesetzt. Wir fordern

$$
\sum_{1}^{N}\left|\xi_{\lambda}^{2}\right|=\text { Min }
$$

unter der Nebenbedingung $\sum_{1}^{n}\left|\tau_{k}^{2}\right|=1$.

8 Vgl. z. B. P. T. Mathews u. A. Salam, Proc. Roy. Soc., Lond. A 221, 128 [1954].
Nach dem Lagrange-Verfahren ergibt sich

$$
\frac{\partial}{\partial \tau_{i}^{*}}\left\{\sum_{1}^{N}\left|a_{\lambda k} \tau_{k}\right|^{2}-Z\left(\sum_{1}^{N}\left|\tau_{k}^{2}\right|-1\right)\right\}=0,
$$

d. h. $\quad \sum_{1}^{N} \sum_{1}^{n} a_{\lambda i}^{*} a_{\lambda k} \tau_{k}-Z \tau_{i}=0$.

Dann ist $\quad Z=Z(E)=\sum_{1}^{N}\left|a_{\lambda k} \tau_{k}\right|^{2}$

die gesuchte Quadratsumme, d. h. der Fehler der Gleichungen; der Energieeigenwert $E$ ist jener Wert von $E$, für den $Z(E)$ den kleinsten Betrag annimmt. Wenn $Z=0$ als Minimum vorkommt, so sind alle Eigenwertgleichungen gleichzeitig exakt erfüllt. Dies wird aber eher die Ausnahme als die Regel sein.

Als einfachstes Beispiel, an dem die charakteristischen Züge und Schwierigkeiten des Verfahrens studiert werden können, seien die beiden Gleichungen $\left[1-f(E)\left(1-\alpha \gamma_{5}\right)\right] \tau=\xi_{1} ; \quad C\left(1+\gamma_{4}\right) \tau=\xi_{2}$

zur Diskussion gestellt, die in etwas allgemeinerer Form bei der Theorie der Bosonen auftreten werden. Die Größen $f(E), \alpha$ und $C$ seien reell. Die Minimalforderung (51) führt zu

$$
\left.\begin{array}{c}
\left\{\left[1-f(E)\left(1-\alpha \gamma_{5}\right)\right]^{2}+2 C^{2}\left(1+\gamma_{4}\right)-Z\right\} \tau=0 ; \\
\left\{[1-f(E)]^{2}+\alpha^{2} f^{2}(E)+2 C^{2}-Z\right\}^{2} \\
=\{2 f(E)(1-f(E)) \cdot \alpha\}^{2}+4 C^{4} ;
\end{array}\right\} \begin{gathered}
Z=[1-f(E)]^{2}+\alpha^{2} f^{2}(E)+2 C^{2} \\
-2 \sqrt{C^{4}+\alpha^{2} f^{2}(E)[1-f(E)]^{2}} .
\end{gathered}
$$

Das Minimum von $Z(E)$ bestimmt den Eigenwert $E$; daher tritt in (57) auch nur das negative Zeichen der Wurzel auf.

Wenn $C^{2} \ll \alpha f(E)$ ist, so gibt es zwei Minima von $Z(E)$, die bei $f(E) \approx 1 /(1+\alpha)$ und $f(E) \approx 1 /(1-\alpha)$ liegen. Der Fehler $Z(E)$ wird an diesen Stellen:

$$
\begin{aligned}
& Z \sim 2 C^{2}-\frac{C^{4}}{a f(E)|(1-f(E))|}, \\
& Z=2 C^{2}-C^{4} \frac{(1+a)^{2}}{a^{2}} \text { für } f(E)=\frac{1}{1+a}, \\
& Z=2 C^{2}-C^{4} \frac{(1-\alpha)^{2}}{\alpha^{2}} \text { für } f(E)=\frac{1}{1-\alpha} .
\end{aligned}
$$

Der Fehler ist also für $\alpha>0$ geringer an der Stelle $f(E)=1 /(1+\alpha)$, er verschwindet für beide Stellen im Grenzfall $C \rightarrow 0$. Für hinreichend kleine Werte von $C$ wird man also $z w e i$ Eigenwerte $f(E)=1 /(1+\alpha)$

๑ Eine ähnliche Vorschrift wurde auch von K. Ladányi, Z. Naturforschg. 16 a, 79 [1961], angegeben. 
und $f(E)=1 /(1-\alpha)$ annehmen müssen. Wenn jedoch $C^{2} \gg \alpha f(E)[1-f(E)]$ ist, so gibt es nur noch ein Minimum von $Z$, das an der Stelle $f(E)=1 /\left(1+\alpha^{2}\right)$ liegt. Dort wird

$$
Z \approx \frac{a^{2}}{1+a^{2}}-\frac{a^{6}}{C^{2}\left(1+a^{2}\right)^{4}} .
$$

Die Frage, ob es einen oder zwei Eigenwerte gibt, hängt also von dem Gewicht $C \mathrm{ab}$, mit dem die Gleichung $C\left(1+\gamma_{4}\right) \tau=0$ bewertet wird. Aber selbst bei einem kleinen Wert von $C$ wird man noch zweifeln müssen, ob auch $f(E)=1 /(1-\alpha)$ ein echter Eigenwert ist, da hier der Fehler $Z$ größer ist als für $f(E)=1 /(1+\alpha)$.

Man erkennt daraus, da $\beta$ das hier vorgeschlagene modifizierte TAMM-DANCofF-Verfahren oft keine eindeutige Entscheidung darüber zuläßt, ob ein gefundenes Minimum von $Z(E)$ zu einem echten Eigenwert gehört, da man eben kein quantitatives Kriterium dafür angeben kann, ein wie großer Fehler in den durch Weglassen gewisser $\varphi$-Funktionen gewonnenen $\mathrm{T}_{\text {AMM }}-\mathrm{D}_{\text {ANCOFF-Gleichungen noch zugelassen }}$ werden darf. Besonders kompliziert wird es, wenn der Gewichtsfaktor $C$ selbst noch von $E$ abhängt. Trotzdem wird man diesen Zug des Verfahrens kaum als einen Mangel gegenüber der üblichen Methode ansehen können, da er nur eine allgemeine Schwäche des TAMM-DAncofF-Verfahrens deutlich macht, die bei der üblichen Methode verschleiert wird.

Die im vorliegenden Abschnitt etwas schematisch geschilderte Methode soll im folgenden bei der praktischen Anwendung auf die Fermionen und später auf die Bosonen noch ausführlicher begründet und im einzelnen durchgeführt werden.

\section{b) Ableitung der Eigenwertgleichungen}

1. Eigenwertgleichungen der Fermionen

Die Eigenwertgleichung der Fermionen soll in einer Näherung bestimmt werden, in der in einem

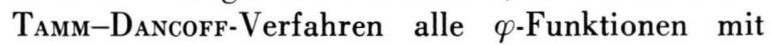
mehr als drei Variabeln vernachlässigt werden. Außerdem sollen die 3-Punktfunktionen $\varphi(x y \mid z)$ der FERMI-Teilchen für verschiedene Koordinaten durch zusätzliche Forderungen mit den entsprechenden 3-Punktfunktionen für gleiche Koordinaten in Beziehung gesetzt werden. Es reicht in diesem Fall dann aus, das gekoppelte Gleichungssystem zu betrachten, das man durch Iteration der Einpunktfunk- tion $\varphi(\underset{\alpha}{x})$ und der 3-Punktfunktion mit gleichen Koordinaten $\varphi \underset{\varrho}{(\underset{\sigma}{x} \boldsymbol{x} \mid \underset{\tau}{x})}$ erhält. Hier sei zur Klärung der Bezeichnungsweise festgestellt, daß für die zur Entstehung eines Fermions gehörigen Einpunktfunktionen $\tau(\underset{\alpha}{x})=\varphi \underset{\alpha}{(x)}$ gesetzt werden kann, ebenso für die zur Entstehung von Bosonen gehörigen Zweipunktfunktionen

$$
\tau(\underset{\alpha \beta}{x} \underset{\beta}{x} \mid)=\varphi(\underset{\alpha \beta}{x} \underset{\beta}{x} \mid) \quad \text { oder } \quad \tau(\underset{\alpha}{x} \mid \underset{\beta}{x})=\varphi(\underset{\alpha}{x} \mid \underset{\beta}{x})
$$

Für die praktische Rechnung erweist es sich als zweckmäßig, die 3-Punktfunktion nach der Basis einer Algebra zu entwickeln, die man als direktes Produkt aus der Dirac- und Isospinalgebra gewinnt:

$$
\varphi(\underset{\varrho \sigma \sigma}{x} \underset{\tau}{x} \mid x)=\sum_{i=1}^{64} \sum_{j=1}^{64}\left(\Gamma_{i}\right)_{\varrho \varkappa}\left(\Gamma_{j}\right)_{\sigma \tau} \varphi_{i j, \varkappa}(x),
$$

wobei die Indizes $\varrho, \varkappa, \sigma, \tau$ hier von 1 bis 8 laufen und die $\Gamma_{i}$ die 64 Basismatrizen in diesem Raum darstellen.

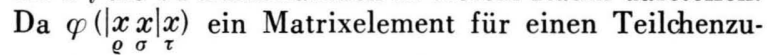
stand mit Spin und Isospin 1/2 sein soll, dürfen in der Doppelsumme nur folgende Glieder der Dirac-IsospinAlgebra auftreten

$$
\begin{aligned}
{\left[1 \times 1, \Gamma_{5} \times \Gamma_{5},\right.} & \Gamma_{\mu} \times \Gamma^{\mu}, \Gamma_{5} \Gamma_{\mu} \times \Gamma_{5} \Gamma^{\mu} \\
& \left.\Gamma_{\mu \nu} \times \Gamma^{\mu \nu}\right]_{\varrho_{1} \varkappa_{1}, \sigma_{1} \tau_{1}} \times[1 \times 1]_{\varrho_{2} \varkappa_{2}, \sigma_{2} \tau_{2}}
\end{aligned}
$$

und

$$
[\ldots \ldots]_{\varrho_{1} \varkappa_{1}, \sigma_{1} \tau_{1}} \times\left[\tau^{l} \times \tau^{l}\right]_{\varrho_{2} \varkappa_{2}, \sigma_{2} \tau_{2}},
$$

wobei die $\varrho_{1} \varkappa_{1} \sigma_{1} \tau_{1}$ je von 1 bis 4 (Dirac-Raum) und die $\varrho_{2} \varkappa_{2} \sigma_{2} \tau_{2}$ je von 1 bis 2 (Isospin-Raum) laufen. Die $\Gamma_{\mu}, \Gamma_{\mu \nu}, \Gamma_{5}$ sind die üblichen Matrizen im DiracSpinraum. Anstatt im Isospinraum die Basiselemente (63) mit $\tau^{l} \times \tau^{l}$ zu betrachten, kann man auch die Basiselemente

$$
[\ldots \ldots]_{\sigma_{1} \varkappa_{1}, \varrho_{1} \tau_{1}} \times[1 \times 1]_{\sigma_{2} \varkappa_{2}, \varrho_{2} \tau_{2}}
$$

verwenden, die durch FiERz-Transformationen aus (62) und (63) hervorgehen und sich von den Basiselementen (62) nur durch die Vertauschung von $\varrho$ und $\sigma$ unterscheiden. Da die $\varphi$-Funktionen jedoch bezüglich der durch $\varrho$ und $\sigma$ indizierten Feldoperatoren antisymmetrisch sind, lassen sich diese 5 Elemente auf die 5 Elemente (62) zurückführen.

Im Falle der 3-Punkt-Matrixelemente der FERMITeilchen brauchen wir also im folgenden nur die 5 speziellen Projektionen zu berücksichtigen:

$$
\begin{aligned}
& \varphi_{1}(\underset{\alpha}{x})=\left(\Gamma_{5} \Gamma_{\mu}\right)_{\alpha_{\varrho}}\left(\Gamma_{5} \Gamma^{\mu}\right)_{\tau \sigma} \varphi(\underset{\varrho}{x} \underset{\tau}{x} \mid \underset{\tau}{x}), \\
& \left.\varphi_{2}(\underset{\alpha}{x})=\left.\left(\Gamma_{\mu}\right)_{\alpha \varrho}\left(\Gamma^{\mu}\right)_{\tau \sigma} \varphi \underset{\varrho}{\left(\left.\underset{\sigma}{x}\right|_{\tau}\right.}\right|_{\tau} ^{x}\right), \\
& \left.\varphi_{3} \underset{\alpha}{(x)}=\left(\Gamma_{5}\right)_{\alpha \varrho}\left(\Gamma_{5}\right)_{\tau \sigma} \varphi \underset{\varrho}{(\underset{\sigma}{x} \underset{\tau}{x} \mid x}\right), \\
& \varphi_{4}(\underset{\alpha}{x})=1_{\alpha \varrho} 1_{\tau \sigma} \varphi(\underset{\varrho \sigma}{x \underset{\tau}{x} \mid x} \mid \underset{\tau}{x}), \\
& \varphi_{5}(\underset{\alpha}{x})=\left(\Gamma_{\mu v}\right)_{\alpha \varrho}\left(\Gamma^{\mu v}\right)_{\tau \sigma} \varphi\left(\left.\underset{\varrho}{x} \underset{\tau}{x}\right|_{\tau} ^{x}\right) .
\end{aligned}
$$

Hierbei soll jede $\Gamma$-Matrix gleichzeitig als Einheitsmatrix im Isospinraum aufgefaßt werden. 
Da alle diese Matrixelemente zu einem Zustand mit dem Gesamtimpuls $J^{\mu}$ gehören sollen, wobei $\sqrt{-J^{2}}=\varkappa$ die Masse des Fermi-Teilchens bedeutet, läßt sich auf Grund der Translationskovarianz die $x$-Abhängigkeit der Matrixelemente abspalten

$$
\left.\varphi_{i} \underset{\alpha}{x}\right)=\varphi_{i, \alpha} \exp \left(i J^{\mu} x_{\mu}\right) .
$$

Dasselbe gilt auch für die Einpunktfunktion

$$
\varphi(\underset{\alpha}{x})=\varphi_{\alpha} \exp \left(i J^{\mu} x_{\mu}\right) .
$$

Die Iteration der Einpunktfunktion $\varphi \underset{\alpha}{x})=\tau(\underset{\alpha}{x})$ liefert auf Grund der Dgl. (7) für den 8-komponentigen Feldoperator $X(\underset{\alpha}{x})$ eine Verknüpfung zwischen der Einpunktfunktion $\varphi\left(x_{\alpha}^{x}\right)$ und den speziellen 3 Punktfunktionen $\varphi_{1}(\underset{\alpha}{x})$ und $\varphi_{2}(\underset{\alpha}{x})$. Nach Abspaltung der Ortsabhängigkeit lautet diese Beziehung

$$
\left(\Gamma_{\mu} J^{\mu}\right)_{\alpha \beta} \varphi_{\beta}=-\frac{i l^{2}}{2}\left(\varphi_{1, \alpha}+\varphi_{2, a}\right) .
$$

Bei Iteration einer 3-Punktfunktion mit gleichen Koordinaten erhalten wir nach der Kontraktion eine integrale Verknüpfung dieser Funktion mit 5-Punktfunktionen, mit 3-Punktfunktionen von verschiedenen Koordinaten und mit der Einpunktfunktion. Zum Beispiel ergibt sich bei Iteration der $\varphi_{1}$-Funktion nach der ersten Koordinate nach Anwendung der Wickschen Regel

$$
\begin{aligned}
& \varphi_{1}(\underset{\alpha}{x})=\frac{i l^{2}}{2} \int \mathrm{d}^{4} x^{\prime}\left\{\Gamma_{5} \Gamma_{\mu} G\left(x-x^{\prime}\right) \Gamma_{5} \Gamma_{\nu} F\left(x^{\prime}-x\right)\right. \\
& \cdot \Gamma_{5} \Gamma^{\mu} F\left(x-x^{\prime}\right) \Gamma_{5} \Gamma^{\nu} \\
& +\Gamma_{5} \Gamma_{\mu} G\left(x-x^{\prime}\right) \Gamma_{\nu} F\left(x^{\prime}-x\right) \Gamma_{5} \Gamma^{\mu} F\left(x-x^{\prime}\right) \Gamma^{\nu} \\
& -\Gamma_{5} \Gamma_{\mu} G\left(x-x^{\prime}\right) \Gamma_{5} \Gamma_{v} \\
& \operatorname{Spur}\left[\Gamma_{5} \Gamma^{\mu} F\left(x-x^{\prime}\right) \Gamma_{5} \Gamma^{v} F\left(x^{\prime}-x\right)\right] \\
& -\Gamma_{5} \Gamma_{\mu} G\left(x-x^{\prime}\right) \Gamma_{v} \\
& \left.\operatorname{Spur}\left[\Gamma_{5} \Gamma^{\mu} F\left(x-x^{\prime}\right) \Gamma^{\nu} F\left(x^{\prime}-x\right)\right]\right\}_{\alpha \beta} \varphi \underset{\beta}{\left(x^{\prime}\right)} \\
& +\frac{i l^{2}}{2} \int \mathrm{d}^{4} x^{\prime}\left\{\left[\Gamma_{5} \Gamma_{\mu} G\left(x-x^{\prime}\right) \Gamma_{5} \Gamma_{\nu}\right]_{\alpha \sigma}\right. \\
& \cdot\left[\Gamma_{5} \Gamma^{v} F\left(x^{\prime}-x\right) \Gamma_{5} \Gamma^{\mu}\right]_{\tau \varrho} \\
& +\left[\Gamma_{5} \Gamma_{u} G\left(x-x^{\prime}\right) \Gamma_{\nu}\right]_{\alpha \sigma}\left[\Gamma^{\nu} F\left(x^{\prime}-x\right) \Gamma_{5} \Gamma^{\mu}\right]_{\imath} \\
& -\left[\Gamma_{5} \Gamma_{\mu} G\left(x-x^{\prime}\right) \Gamma_{5} \Gamma_{v} F\left(x^{\prime}-x\right) \Gamma_{5} \Gamma^{\mu}\right]_{\alpha} \\
& \cdot\left[\Gamma_{5} \Gamma^{v}\right]_{\tau \sigma} \\
& -\left[\Gamma_{5} \Gamma_{u} G\left(x-x^{\prime}\right) \Gamma_{v} F\left(x^{\prime}-x\right) \Gamma_{5} \Gamma^{\mu}\right]_{\alpha \rho} \\
& \left.\cdot\left[\Gamma^{v}\right]_{\tau \sigma}\right\} \varphi\left(\underset{\varrho}{x} x^{\prime} \mid x_{\tau}^{\prime}\right) \\
& -\left\{\left[\Gamma_{5} \Gamma_{\mu} G\left(x-x^{\prime}\right) \Gamma_{5} \Gamma_{v}\right]_{\alpha \rho}\right. \\
& \cdot\left[\Gamma_{5} \Gamma^{\mu} F\left(x-x^{\prime}\right) \Gamma_{5} \Gamma^{\nu}\right]_{\tau \sigma} \\
& +\left[\Gamma_{5} \Gamma_{\mu} G\left(x-x^{\prime}\right) \Gamma_{v}\right]_{\alpha ?} \\
& \text { - } \left.\left[\Gamma_{5} \Gamma^{\mu} F\left(x-x^{\prime}\right) \Gamma^{\nu}\right]_{\tau \sigma}\right\} \varphi \underset{\varrho}{\left(\underset{\sigma}{x^{\prime}} x_{\sigma}^{\prime} \mid x\right)}
\end{aligned}
$$

$$
\begin{aligned}
& +\frac{i l^{2}}{2} \int \mathrm{d}^{4} x^{\prime}\left\{\left[\Gamma_{5} \Gamma_{\mu} G\left(x-x^{\prime}\right) \Gamma_{5} \Gamma_{\nu}\right]_{\alpha \varphi}\right. \\
& \text { - }\left[\Gamma_{5} \Gamma^{\mu}\right]_{\tau \sigma}\left[\Gamma_{5} \Gamma^{\nu}\right]_{\varepsilon \lambda} \\
& \left.+\left[\Gamma_{5} \Gamma_{\mu} G\left(x-x^{\prime}\right) \Gamma_{\nu}\right]_{\alpha \varphi}\left[\Gamma_{5} \Gamma^{\mu}\right]_{\tau \sigma}\left[\Gamma^{\nu}\right]_{\varepsilon \lambda}\right\}
\end{aligned}
$$

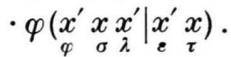

Bei Iteration nach der zweiten und dritten Koordinate erhält man ähnliche Gleichungen. Für die übrigen vier $\varphi$-Funktionen, d.h. $\varphi_{2} \ldots \varphi_{5}$ finden wir entsprechende Gleichungstripel, wenn wir in (69) und den anderen, nicht angegebenen Gleichungen $\Gamma_{5} \Gamma_{\mu} \ldots \Gamma_{5} \Gamma^{\mu}$ durch $\Gamma_{\mu} \ldots \Gamma^{\mu}, \Gamma_{5} \ldots \Gamma_{5}, 1 \ldots 1$, $\Gamma_{\mu \nu} \ldots \Gamma^{\mu \nu}$ ersetzen.

Vernachlässigen wir die Integrale über die 5Punktfunktionen im Vergleich zu den 3-Punkt- und 1-Punktfunktionen, so erhalten wir ein Gleichungssystem von 16 Gleichungen für die 6 unbekannten, nur von der Schwerpunktskoordinate abhängigen Matrixelemente $\varphi(\underset{\alpha}{x}), \varphi_{i}(\underset{\alpha}{x})$ und die 10 unbekannten, auch von einer Relativkoordinate abhängigen Funktionen von der algebraischen Form $\varphi_{1} \ldots \varphi_{5}$, aber den Koordinatenkombinationen $\left(x, x^{\prime} \mid x^{\prime}\right)$ und $\left(x^{\prime} x^{\prime} \mid x\right)$. Das System ist also weitgehend unterbestimmt. Um es bestimmt zu machen, müßten wir auch sämtliche Gleichungen heranziehen, die sich durch Iteration von 3-Punktfunktionen für verschiedene Koordinaten ergeben. Dies würde das Problem jedoch wesentlich komplizierter gestalten.

Statt dessen wollen wir über die in der Gleichung auftretenden 3 -Punktfunktionen $\varphi\left(x x^{\prime} \mid x^{\prime}\right)$ und $\varphi\left(x^{\prime} x^{\prime} \mid x\right)$ speziell verfügen, indem wir bestimmte Ansätze für ihre Abhängigkeit von der Relativkoordinate $\left(x-x^{\prime}\right)$ machen. Nimmt man an, daß diese Funktionen von $x-x^{\prime}$ schnell im Vergleich zu $\varkappa^{-1}$ abfallen, so lassen sich die in den Gln. (69 u. a.) auftretenden Integrale über diese Funktionen im Vergleich zu den 3-Punktfunktionen am gleichen Ort auf der linken Seite dieser Gleichungen vernachlässigen. Diese Annahme wird kaum gut erfüllt sein, da man einen Zusammenhang zwischen der Abhängigkeit der 3-Punktfunktion von der Relativkoordinate und der Ausdehnung des Fermions und deshalb mindestens einen mit der СомртоN-Wellenlänge vergleichbaren Abfall vermuten würde; doch soll hier diese Annahme gemacht werden, um die Rechnungen nicht übermäßig kompliziert werden zu lassen. Um den Einfluß dieser speziellen Annahme auf die Rechnung zu prüfen, soll in einer späteren Arbeit an dieser Stelle die entgegengesetzte und in anderer Hinsicht schlecht erfüllte Voraussetzung gemacht werden, daß die 3-Punktfunktion von der Relativkoordinate über- 
haupt nicht abhängt. Die Rechnungen werden in diesem Fall nur wenig komplizierter. Diese Voraussetzung lag einer von Just, Hartmann und v. Ossowski (s. Anm. ${ }^{10}$ ) durchgeführten Berechnung der Nukleonenmasse zugrunde, die nur eine sehr kleine Korrektur gegenüber den Rechnungen in den früheren Arbeiten ergab, in denen die 3-Punktfunktionen $\mathrm{zu}$ verschiedenen Koordinaten vernachlässigt wurden. Es ist daher zu erwarten, daß die Berücksichtigung dieser Funktionen auch in dem hier durchgeführten allgemeineren Verfahren die Rechnungen nicht wesentlich verändern würde.

Jegliche spezielle Annahme über die 3-Punktfunktion $\mathrm{zu}$ verschiedenen Koordinaten führt im allgemeinen dazu, daß das resultierende approximative Gleichungssystem in dem in II a) erörterten Sinne überbestimmt wird und deshalb im allgemeinen nicht mehr streng zu erfüllen ist. Die Überbestimmung kann dann zu einem gewissen Grade benützt werden, um die Verträglichkeit der an verschiedenen Stellen vorgenommenen Näherungen abzuschätzen.

Vernachlässigt man die Matrixelemente mit ungleichen Koordinaten und separiert den Gesamtimpuls ab, so haben die Gln. (69 u. a.) im Impulsraum die Form

$$
\varphi_{i, \alpha}^{j}(J)=\frac{i \varkappa^{6} l^{2}}{2(2 \pi)^{8}} \int \frac{\mathrm{d}^{4} q \mathrm{~d}^{4} r}{N(q, r, J)} M_{i, \alpha}^{j}(q, r, J) .
$$

$\varphi_{i, \alpha}^{j}(J)$ bedeutet hierbei den schwerpunktsunabhängigen Anteil der 3-Punktfunktionen $\varphi_{i}(\underset{\alpha}{(x)}$ in einer Näherung, in der nach der Koordinate $j(j=1,2,3)$ iteriert wurde. Die $M_{i, \alpha}^{j}(q, r, J)$ enthalten in der hier betrachteten Näherung nur die 1-Punktfunktion als Faktor und sind im Falle der $\varphi_{1}, a^{-}$-Matrixelemente durch folgende Ausdrücke gegeben

$$
\begin{aligned}
& M_{1, \alpha}^{1}=\left\{-\Gamma_{5} \Gamma_{\mu}(\Gamma, q-r+J) \Gamma_{5} \Gamma_{\nu} \mathrm{Y}(q)\right. \\
& \cdot \Gamma_{5} \Gamma^{\mu} Y(r) \Gamma_{5} \Gamma^{\nu} \\
& -\Gamma_{5} \Gamma_{\mu}(\Gamma, q-r+J) \Gamma_{\nu} Y(q) \\
& \text { - } \Gamma_{5} \Gamma^{\mu} Y(r) \Gamma^{\nu} \\
& +\Gamma_{5} \Gamma_{\mu}(\Gamma, q-r+J) \Gamma_{5} \Gamma_{v} \\
& \operatorname{Spur}\left[\Gamma_{5} \Gamma^{\mu} Y(r) \Gamma_{5} \Gamma^{v} Y(q)\right] \\
& +\Gamma_{5} \Gamma_{\mu}(\Gamma, q-r+J) \Gamma_{v} \\
& \left.\left.\operatorname{Spur}\left[\Gamma_{5} \Gamma^{\mu} Y(\mathrm{r}) \Gamma^{\nu} Y(q)\right]\right\}\right\}_{\alpha \beta} \varphi_{\beta}, \\
& M_{1, \alpha}^{2}=\left\{-\Gamma_{5} \Gamma_{\mu} Y(r) \Gamma_{5} \Gamma_{v} Y(q)\right. \\
& \text { - } \Gamma_{5} \Gamma^{\mu}(\Gamma, q-r+J) \Gamma_{5} \Gamma^{v} \\
& -\Gamma_{5} \Gamma_{\mu} Y(r) \Gamma_{v} Y(q) \\
& \text { - } \Gamma_{5} \Gamma^{\mu}(\Gamma, q-r+J) \Gamma^{\nu} \\
& +\Gamma_{5} \Gamma_{\mu} Y(r) \Gamma_{5} \Gamma_{\nu} \\
& \operatorname{Spur}\left[\Gamma_{5} \Gamma^{\mu}(\Gamma, q-r+J) \Gamma_{5} \Gamma^{\nu} Y(q)\right] \\
& +\Gamma_{5} \Gamma_{\mu} Y(r) \Gamma_{\nu} \\
& \left.\left.\operatorname{Spur}\left[\Gamma_{5} \Gamma^{\mu}(\Gamma, q-r+J) \Gamma^{\nu} Y(q)\right]\right\}\right\}_{\alpha \beta} \varphi_{\beta},
\end{aligned}
$$

$$
\begin{aligned}
M_{1, \alpha}^{3}=\{ & \Gamma_{5} \Gamma_{\mu} Y(-q) \Gamma_{5} \Gamma_{v}(\Gamma, q-r+J) \\
& \cdot \Gamma_{5} \Gamma^{\mu} Y(r) \Gamma_{5} \Gamma^{v} \\
& +\Gamma_{5} \Gamma_{\mu} Y(-q) \Gamma_{v}(\Gamma, q-r+J) \\
& \cdot \Gamma_{5} \Gamma^{\mu} Y(r) \Gamma^{v} \\
& -\Gamma_{5} \Gamma_{\mu} Y(-q) \Gamma_{5} \Gamma_{v} \\
& \quad \operatorname{Spur}\left[\Gamma_{5} \Gamma^{\mu} Y(r) \Gamma_{5} \Gamma^{v}(\Gamma, q-r+J)\right] \\
- & \Gamma_{5} \Gamma_{\mu} Y(-q) \Gamma_{v} \\
& \left.\quad \operatorname{Spur}\left[\Gamma_{5} \Gamma^{\mu} Y(r) \Gamma^{v}(\Gamma, q-r+J)\right]\right\}_{\alpha \beta} \varphi_{\beta} .
\end{aligned}
$$

Die $M$-Funktionen der übrigen Matrixelemente ergeben sich wieder durch die Ersetzung von $\Gamma_{5} \Gamma_{\mu} \ldots \Gamma_{5} \Gamma^{\mu}$ durch $\Gamma_{\mu} \ldots \Gamma^{\mu}$ etc.

Die Funktion $N(q, r, J)$ im Nenner des Integranden von (70) hat die Bedeutung

$N(q, r, J)=(q-r+J)^{2}\left(q^{2}+\varkappa^{2}\right)\left(r^{2}+\varkappa^{2}\right)\left(q^{2}\right)\left(r^{2}\right)$,

wobei über die Pole im Feynmanschen Sinn zu integrieren ist. Für die Greensche Funktion $G(x)$ und die Kontraktionsfunktion $F(x)$ wurde wieder gesetzt

$$
\begin{aligned}
& G(x)=-\frac{1}{(2 \pi)^{4}} \int \mathrm{d}^{4} p e^{i p x} \frac{\Gamma_{\mu} p^{\mu}}{p^{2}}, \\
& F(x)=\frac{\varkappa^{3}}{(2 \pi)^{4}} \int \mathrm{d}^{4} p e^{i p x} \frac{Y(p)}{p^{2}\left(p^{2}+\varkappa^{2}\right)}
\end{aligned}
$$

mit der Abkürzung

$$
Y(p)=-\frac{\Gamma_{\mu} p^{\mu} \varkappa}{p^{2}} Z_{p}+i Z_{\varkappa} .
$$

Bei der speziellen Wahl $Z_{p}=Z_{\kappa}=1$ entspricht $F(x)$ der in den früheren Arbeiten "A“ (Abs. IV d) und „B“ verwendeten Kontraktionsfunktion. Wir müssen diese Kontraktionsfunktion, um die Beschreibung der seltsamen Teilchen einbeziehen zu können, dahingehend verallgemeinern, daß wir für $Z_{\mathrm{p}}$ und $Z_{\varkappa}$ auch eine $\mathrm{Ab}$ hängigkeit von $\Gamma_{5}$ und $\tau$ und den auf den Grundzustand wirkenden Operatoren $\varrho, \Sigma$ zulassen. Wir wollen diese Abhängigkeit vorläufig noch ganz allgemein lassen, da die Rechnungen dadurch nur wenig komplizierter werden, und erst am Schluß den im Abschnitt Id) geforderten speziellen Ansatz machen. Folgende Bezeichnungsweise erweist sich dabei als nützlich:

$$
\begin{array}{ll}
Z_{p}=Z_{1}^{p}+\Gamma_{5} Z_{2}^{p}, & Z_{p}{ }^{\prime}=Z_{1}^{p}-\Gamma_{5} Z_{2}^{p}, \\
Z_{\varkappa}=Z_{1}{ }^{\varkappa}+\Gamma_{5} Z_{2}{ }^{\varkappa}, & Z_{\varkappa}{ }^{\prime}=Z_{1}{ }^{\varkappa}-\Gamma_{5} Z_{2}{ }^{\varkappa}
\end{array}
$$

und ferner

$$
Z_{p}{ }^{ \pm}=Z_{1}{ }^{p} \pm Z_{2}{ }^{p}, \quad Z_{\varkappa}{ }^{ \pm}=Z_{1}{ }^{*} \pm Z_{2}{ }^{*} .
$$

Die Größen $Z_{i}^{p}, Z_{i}^{*}$ sollen dann noch von den Isospinmatrizen und den Operatoren $\varrho, \Sigma$ abhängen.

Auch ist es zweckmäßig, bei der Berechnung des Gleichungssystems wieder die schon früher in "A " berechneten dimensionslosen Funktionen $L(X), M(X)$ und $N(X)$ einzuführen, die nur noch von der dimen.

10 K. Just, J. Hartmann u. H. v. Ossowski, Z. Phys. 158, 39 [1960]. 
sionslosen Größe $X=\sqrt{\sqrt{J^{2}} \mid / \varkappa^{2}}$ abhängen.

$$
\begin{aligned}
J_{u} L(X) & =-\frac{\varkappa^{4}}{\pi^{4}} \int \mathrm{d}^{4} q \mathrm{~d}^{4} r \frac{(q r)(q-r+J)_{\mu}}{N(q, r, J) q^{2} r^{2}} \\
& =-\frac{\varkappa^{4}}{\pi^{4}} \int \mathrm{d}^{4} q \mathrm{~d}^{4} r \frac{r_{\mu}(q, q-r+J)}{N(q, r, J) q^{2} r^{2}}, \\
J_{u} M(X) & =-\frac{\varkappa^{2}}{\pi^{4}} \int \mathrm{d}^{4} q \mathrm{~d}^{4} r \frac{(q-r+J) \mu}{N(q, r, J)}, \\
N(X) & =-\frac{\varkappa^{2}}{\pi^{4}} \int \mathrm{d}^{4} q \mathrm{~d}^{4} r \frac{(q, q-r+J)}{N(q, r, J) q^{2}} .
\end{aligned}
$$

Die 15 Gleichungen für die konstanten Spinoren $\varphi_{i, a}$ lassen sich dann alle auf die Form bringen

$$
\begin{gathered}
\varphi_{i, \alpha}^{j}=-\frac{i}{l^{2}}\left(\frac{\varkappa l}{4 \pi}\right)^{4}\left\{\left[c_{L, i}^{j} L(X)-c_{M, i}^{j} M(X)\right] \Gamma_{\mu} J^{\mu}\right. \\
\left.-i \varkappa c_{N, i}^{j} N(X)\right\}_{\alpha \beta} \varphi_{\beta} .
\end{gathered}
$$

Es ist für das Folgende jedoch günstiger und dem
Problem viel angemessener, nicht die fünf 3-Punktfunktionen $\varphi_{i, a}$ zu betrachten, sondern die bezüglich der Helizität (oder dem Vorzeichen von $l$ ) diagonalilisierten speziellen Linearkombinationen

$$
\begin{aligned}
& \overline{\varphi_{1}}=\frac{1}{2}\left(\varphi_{1}+\varphi_{2}\right), \overline{\varphi_{2}}=\frac{1}{2}\left(\varphi_{1}-\varphi_{2}\right), \\
& \overline{\varphi_{3}}=\frac{1}{2}\left(\varphi_{3}+\varphi_{4}\right), \overline{\varphi_{4}}=\frac{1}{2}\left(\varphi_{3}-\varphi_{4}\right), \overline{\varphi_{5}}=\varphi_{5} .
\end{aligned}
$$

Auch für diese Spinoren $\bar{\varphi}, a$ gelten dann wieder Gleichungen derselben Form

$$
\bar{\varphi}_{i, \alpha}^{j}=-\frac{i}{l^{2}}\left(\frac{\varkappa l}{4 \pi}\right)^{4}\left\{\left[\bar{c}_{L, i}^{j} L(X)-\bar{c}_{M, i}^{j} M(X)\right] \Gamma_{\mu} J^{\mu}\right.
$$$$
\left.-i \varkappa \bar{c}_{N, i}^{j} N(X)\right\}_{\alpha \beta} \varphi_{\beta} .
$$

Die Beziehung (8) für die 1-Punktfunktion lautet jetzt einfach

$$
\left(\Gamma_{\mu} J^{\mu}\right)_{\alpha \beta} \varphi_{\beta}=-\left(i l^{2}\right) \bar{\varphi}_{1, \alpha} .
$$

Für die 45 Koeffizienten $\bar{c}_{L, i}^{j}, \bar{c}_{M, i}^{j}$ und $\bar{c}_{N, i}^{j}$ ergibt sich nach einiger Rechnung

$\bar{c}_{L, 1}^{1}=8\left[Z_{p^{2}}+\mathrm{Sp}\left(Z_{1} p^{2}+Z_{2}^{p^{2}}\right)+\Gamma_{5} \operatorname{Sp}\left(Z_{1}^{p} Z_{2}^{p}-Z_{2}{ }^{p} Z_{1}^{p}\right)\right]$,

$\vec{c}_{L, 1}^{2}=\bar{c}_{L, 1}^{3}=8\left[Z_{p}^{2}+Z_{p} \operatorname{Sp} Z_{1}^{p}+\Gamma_{5} Z_{p} \operatorname{Sp}_{2}{ }^{p}\right]$,

$\bar{c}_{M, 1}^{1}=\bar{c}_{M, 1}^{2}=\bar{c}_{M, 1}^{3}=\bar{c}_{N, 1}^{1}=\bar{c}_{N, 1}^{2}=\bar{c}_{N, 1}^{3}=0 ;$

$\bar{c}_{L, 2}^{1}=\bar{c}_{L, 2}^{2}=\bar{c}_{L, 2}^{3}=\bar{c}_{M, 2}^{2}=\bar{c}_{M, 2}^{3}=\bar{c}_{N, 2}^{1}=0, \quad \bar{c}_{M, 2}^{1}=4\left[Z_{\varkappa}{ }^{\prime} Z_{\varkappa}+\operatorname{Sp}\left(Z_{1}{ }^{{ }^{2}}-Z_{2}{ }^{{ }^{2}}\right)-\Gamma_{5} \operatorname{Sp}\left(Z_{1}{ }^{*} Z_{2}{ }^{*}-Z_{2}{ }^{*} Z_{1}{ }^{*}\right)\right]$,

$\bar{c}_{N, 2}^{2}=\bar{c}_{N, 2}^{3}=4\left[Z_{\varkappa}{ }^{\prime} Z_{p}{ }^{\prime}+Z_{\varkappa}{ }^{\prime} \mathrm{Sp} Z_{1}{ }^{p}-\Gamma_{5} Z_{\varkappa}{ }^{\prime} \mathrm{Sp} Z_{2}{ }^{p}\right]$;

$\bar{c}_{L, 3}^{1}=\bar{c}_{L, 3}^{2}=\bar{c}_{L, 3}^{3}=\bar{c}_{M, 3}^{1}=\bar{c}_{M, 3}^{2}=\bar{c}_{N, 3}^{3}=0$,

$\bar{c}_{N, 3}^{1}=-2\left[Z_{\varkappa} Z_{p}{ }^{\prime}+\operatorname{Sp}\left(Z_{1}{ }^{*} Z_{1}{ }^{p}-Z_{2}{ }^{*} Z_{2}{ }^{p}\right)-\Gamma_{5} \operatorname{Sp}\left(Z_{1}{ }^{*} Z_{2}{ }^{p}-Z_{2}{ }^{*} Z_{1}{ }^{p}\right)\right]$,

$\bar{c}_{N, 3}^{2}=-2\left[Z_{\mathrm{p}}{ }^{\prime} Z_{\varkappa}+Z_{p}{ }^{\prime} \operatorname{Sp} Z_{1}{ }^{*}+\Gamma_{5} Z_{p}{ }^{\prime} \operatorname{Sp} Z_{2}{ }^{*}\right]$,

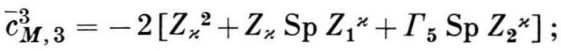

$\bar{c}_{L, 4}^{1}=\bar{c}_{L, 4}^{2}=\bar{c}_{L, 4}^{3}=\bar{c}_{M, 4}^{1}=\bar{c}_{M, 4}^{3}=\bar{c}_{N, 4}^{2}=0$,

$\bar{c}_{N, 4}^{1}=-2\left[Z_{p}{ }^{\prime} Z_{\varkappa}{ }^{\prime}+\operatorname{Sp}\left(Z_{1}{ }^{p} Z_{1}{ }^{\alpha}+Z_{2}{ }^{p} Z_{2}{ }^{\kappa}\right)-\Gamma_{5} \mathrm{Sp}\left(Z_{1}{ }^{p} Z_{2}{ }^{\kappa}+Z_{2}{ }^{p} Z_{1}{ }^{\alpha}\right)\right]$,

$\bar{c}_{M, 4}^{2}=-2\left[Z_{\varkappa} Z_{\varkappa}{ }^{\prime}+Z_{\varkappa} \operatorname{Sp} Z_{1}{ }^{\varkappa}-\Gamma_{5} Z_{\varkappa} \operatorname{Sp} Z_{2}{ }^{\varkappa}\right]$,

$\bar{c}_{N, 4}^{3}=-2\left[Z_{p}^{\prime} Z_{\varkappa}^{\prime}+Z_{p}^{\prime} \operatorname{Sp} Z_{1}{ }^{x}-\Gamma_{5} Z_{p}^{\prime} \operatorname{Sp} Z_{2}{ }^{x}\right] ;$

$\bar{c}_{L, 5}^{1}=\bar{c}_{L, 5}^{2}=\bar{c}_{L, 5}^{3}=\bar{c}_{M, 5}^{1}=0$,

$\bar{c}_{N, 5}^{1}=12\left[2 Z_{\varkappa} Z_{p}{ }^{\prime}+\operatorname{Sp}\left(Z_{1}{ }^{\star} Z_{1}{ }^{p}+Z_{1}{ }^{p} Z_{1}{ }^{\alpha}+Z_{2}{ }^{*} Z_{2}{ }^{p}-Z_{2}{ }^{p} Z_{2}{ }^{\alpha}\right)-\Gamma_{5} \operatorname{Sp}\left(Z_{1}{ }^{\star} Z_{2}{ }^{p}+Z_{2}{ }^{p} Z_{1}{ }^{\alpha}+Z_{2}{ }^{\star} Z_{1}{ }^{p}-Z_{1}{ }^{p} Z_{2}{ }^{\kappa}\right)\right]$,

$\bar{c}_{M, 5}^{2}=12\left[Z_{\varkappa} \operatorname{Sp} Z_{1}{ }^{\varkappa}-\Gamma_{5} Z_{\varkappa} \operatorname{Sp} Z_{2}{ }^{\varkappa}\right], \quad \bar{c}_{N, 5}^{2}=12\left[2 Z_{p}^{\prime} Z_{\varkappa}+Z_{\mathrm{p}}{ }^{\prime} \operatorname{Sp} Z_{1}{ }^{*}+\Gamma_{5} Z_{p}{ }^{\prime} \operatorname{Sp} Z_{2}{ }^{*}\right]$,

$\bar{c}_{M, 5}^{3}=12\left[2 Z_{\varkappa}{ }^{2}+Z_{\varkappa} \operatorname{Sp} Z_{1}{ }^{*}+\Gamma_{5} Z_{\varkappa} \operatorname{Sp} Z_{2}{ }^{\varkappa}\right], \quad \bar{c}_{N, 5}^{3}=12\left[Z_{p}{ }^{\prime} \operatorname{Sp} Z_{1}{ }^{*}-\Gamma_{5} Z_{p}{ }^{\prime} \operatorname{Sp} Z_{2}{ }^{\varkappa}\right]$.

Hierbei bedeutet Sp eine Spur nur im Isospinraum ( $\tau$-Raum); auf die Reihenfolge der Faktoren muß bei der Auswertung von $(87-91)$ geachtet werden.

Die Gln. (85) stellen zusammen mit (86) 16 Gleichungen für die 6 unbekannten konstanten Spinoren $\varphi_{a}$ und $\varphi_{i, a}$ dar. Sie sind nur dann miteinander verträglich, wenn die zehn Bedingungen

$$
\varphi_{i, \alpha}^{1}=\varphi_{i, \alpha}^{2}=\varphi_{i, \alpha}^{3}
$$

erfüllt sind, d. h. wenn die 3-Punktfunktionen von der speziellen Näherung (Iteration der 1., 2. oder 3. Koordinate) unabhängig sind. Dies führt auf die folgenden acht verschiedenen Bedingungsgleichun-

gen, die für das spezielle $J^{\mu}$ des Fermions $\left(J^{2}=-\varkappa^{2}\right)$ erfüllt sein müssen:

$$
\begin{aligned}
& \left(\frac{\varkappa l}{4 \pi}\right)^{4}\left(\bar{c}_{L, 1}^{1}-\bar{c}_{L, 1}^{2}\right) L(X)(\Gamma J) \varphi=0, \\
& \left(\begin{array}{c}
\varkappa l \\
4 \pi
\end{array}\right)^{4}\left[\bar{c}_{M, 2}^{1} M(X)(\Gamma J)-i \varkappa \bar{c}_{N, 2}^{2} N(X)\right) \varphi=0, \\
& \left(\frac{\varkappa l}{4 \pi}\right)^{4}\left(\bar{c}_{N, 3}^{1}-\bar{c}_{N, 3}^{2}\right) i \varkappa N(X) \varphi=0, \\
& \left(\frac{\varkappa l}{4 \pi}\right)^{4}\left[\bar{c}_{M, 3}^{3} M(X)(\Gamma J)-i \varkappa \bar{c}_{N, 3}^{1} N(X)\right] \varphi=0,
\end{aligned}
$$




$$
\begin{aligned}
& \left(\frac{\varkappa l}{4 \pi}\right)^{4}\left(\bar{c}_{N, 4}^{1}-\bar{c}_{N, 4}^{3}\right) i \varkappa N(X) \varphi=0, \\
& \left(\frac{\varkappa l}{4 \pi}\right)^{4}\left[\bar{c}_{M, 4}^{2} M(X)(\Gamma J)-i \varkappa \bar{c}_{N, 4}^{1} N(X)\right] \varphi=0, \\
& \left(\frac{\varkappa l}{4 \pi}\right)^{4}\left[\bar{c}_{M, 5}^{2} M(X)(\Gamma J)\right.
\end{aligned}
$$$$
\left.-i \varkappa\left(\bar{c}_{N, 5}^{1}-\bar{c}_{N, 5}^{2}\right) N(X)\right] \varphi=0,
$$$$
\left(\frac{\varkappa l}{4 \pi}\right)^{4}\left[\left(\bar{c}_{M, 5}^{2}-\bar{c}_{M, 5}^{3}\right) M(X)(\Gamma J)\right.
$$$$
\left.+i \varkappa\left(\bar{c}_{N, 5}^{2}-\bar{c}_{N, 5}^{3}\right) N(X)\right] \varphi=0 .
$$

Durch Kombination von (86) mit der Gl. für $\bar{\varphi}_{1, \alpha}^{1}$ in (85) erhalten wir schließlich noch eine Beziehung, die wir als Eigenwertbedingung bezeichnen wollen

$$
\left[1+\left(\frac{x l}{4 \pi}\right)^{4} \bar{c}_{L, 1}^{1} L(X)\right](\Gamma J) \varphi=0 .
$$

Um uns diese Gleichungen etwas zu veranschaulichen, wählen wir als Beispiel den in der Arbeit „B“ diskutierten Fall mit $Z_{p}=Z_{x}=1$ (keine Vakuumentartung). Dann erhalten wir für die von Null verschiedenen Koeffizienten

$$
\begin{aligned}
& \bar{c}_{L, 1}^{1}=\bar{c}_{L, 1}^{2}=\bar{c}_{L, 1}^{3}=24, \\
& \bar{c}_{M, 2}^{1}=\bar{c}_{N, 2}^{2}=\bar{c}_{N, 2}^{3}=12, \\
& \bar{c}_{N, 3}^{1}=\bar{c}_{N, 3}^{2}=\bar{c}_{M, 3}^{3}=-6, \\
& \bar{c}_{N, 4}^{1}=\bar{c}_{M, 4}^{2}=\bar{c}_{N, 4}^{3}=-6, \\
& \bar{c}_{N, 5}^{1}=72, \quad \bar{c}_{M, 5}^{2}=\bar{c}_{N, 5}^{3}=24, \\
& \bar{c}_{N, 5}^{2}=\bar{c}_{M, 5}^{3}=48 .
\end{aligned}
$$

Die 8 Gln. $(93-100)$ reduzieren sich, was vom gruppentheoretischen Gesichtspunkt aus nicht erstaunlich ist, auf eine einzige Gleichung

$$
[M(X)(\Gamma J)-i \varkappa N(X)] \varphi=0,
$$

die noch mit der aus Gl. (101) folgenden Beziehung

$$
\left[1+\left(\frac{x l}{4 \pi}\right)^{4} \cdot 24 L(X)\right](\Gamma J) \varphi=0
$$

verträglich sein soll. Die Gl. (104) ist die schon früher aufgetretene Eigenwertgleichung zur Bestimmung der Masse des Fermions. Die Gl. (103) stellt eine Zusatzbedingung an die Lösungen dieser Eigenwertgleichung dar. Im allgemeinen wird man beide Gleichungen zusammen nur approximativ befriedigen können. So folgt in diesem Fall, daß nur die Lösungen der Gl. (104) mit positiver Eigenparität - d. h. Lösungen einer Dirac-Gleichung der Form $[(\Gamma J)-i \varkappa] \varphi=0$ - auch die Gl. (103) näherungsweise erfüllen, da die Funktionen $M$ und $N$ beide positiv sind und in der Nähe von $J^{2}=-\varkappa^{2}$ einen Schnittpunkt haben. Man erhält also nur wieder die in der Kontraktionsfunktion ur- sprünglich angesetzten Nukleonen positiver Parität und damit keine Verdopplung der Lösungsmannigfaltigkeit gegenüber den Eigenwertberechnungen mit der ursprünglichen (die Parität nicht darstellenden) Feldgleichung (1) bzw. (3) mit 4-komponentigen Feldoperatoren. Es besteht demnach eine gewisse Äquivalenz zwischen den beiden Verfahren (mit und ohne Paritätsdarstellung) im Falle der Eigenwerte der Fermionen. Allerdings werden in der paritätssymmetrischen Formulierung, wie man leicht an dem Gleichungspaar $(103,104)$ feststellen kann, bei unserem jetzigen Ansatz die Lösungen zur Masse Null noch unterdrückt. Das ist jedoch nicht erstaunlich, da auch die früher definierte „Parität 2. Art“ für Teilchen der Masse Null nicht existiert.

Im Abschnitt II c) sollen für Fermionen Eigenwertgleichungen unter der Voraussetzung des in I c) besprochenen entarteten Vakuums diskutiert werden. Dann ist $Z_{p}$ und $Z_{\varkappa}$ nicht mehr einfach gleich eins und wir müssen bei dieser Diskusion auf das allgemeinere Gleichungssystem $(93-100)$ mit den Koeffizienten (87 bis 91) zurückgreifen. Die Kontraktionsfunktion (145) in II c) führt zu

$$
Z_{p}=1+a \Gamma_{5} \Sigma_{2} ; \quad Z_{\varkappa}=\beta\left[1+i \eta \Gamma_{5} \Sigma_{3}(\varrho \tau)\right] .
$$

Bei der Multiplikation solcher Ausdrücke ist darauf zu achten, daß im Raum der Grundzustände, also bei den Operatoren $\Sigma$, $\varrho$ nach I d) stets die symmetrisierte Produktbildung gemeint ist. Daher erhält man z. B. für das Produkt $Z_{p} \cdot Z_{\varkappa}$ :

$$
Z_{p} \cdot Z_{\varkappa}=\beta\left[1+\alpha \Gamma_{5} \Sigma_{2}+i \eta \Gamma_{5} \Sigma_{3}(\varrho \tau)\right] .
$$

Dadurch vereinfachen sich die entstehenden Ausdrücke beträchtlich und man kann die den Gln. (103, 104) entsprechenden Formeln ohne Schwierigkeit angeben.

\section{Eigenwertgleichung der Bosonen}

Die Eigenwertgleichung der Bosonen mit der Quantenzahl $I_{\mathrm{N}}=0$ soll in einer Näherung bestimmt werden, in der alle $\varphi$-Funktionen der Bosonen mit mehr als zwei Koordinaten vernachlässigt werden. Diese Näherung ist noch etwas schlechter als die Näherung bei den Fermionen. Wir brauchen hier nur, wie schon in den früheren Arbeiten, Gleichungen zu untersuchen, die sich bei Iteration der 2 Punktfunktion mit gleichen Koordinaten $\varphi(x \mid x)$ ergeben. Wir wollen nun allerdings eine Kontraktionsfunktion von der allgemeinen Form (76) und (77) fordern und die beiden Näherungen, die wir bei Iteration der ersten bzw. zweiten Koordinate erhalten, getrennt ausführen, anstatt gleich über diese zu mitteln.

Bei Iteration der $\varphi$-Funktion nach der ersten Koordinate erhält man nach Kontraktion

$$
\begin{aligned}
& \varphi(\underset{\alpha}{x} \mid x)=\frac{i l^{2}}{2} \int \mathrm{d}^{4} x^{\prime}\left\{\left[G\left(x-x^{\prime}\right) \Gamma_{5} \Gamma_{\nu} F\left(x^{\prime}-x\right)\right]_{\alpha \beta}\left[\Gamma_{5} \Gamma^{\nu}\right]_{\tau \sigma}+\left[G\left(x-x^{\prime}\right) \Gamma_{\nu} F\left(x^{\prime}-x\right)\right]_{\alpha \beta} \Gamma_{\tau^{\nu} \sigma}\right. \\
& \left.-\left[G\left(x-x^{\prime}\right) \Gamma_{5} \Gamma_{\nu}\right]_{a \sigma}\left[\Gamma_{5} \Gamma^{v} F\left(x-x^{\prime}\right)\right]_{\tau \beta}-\left[G\left(x-x^{\prime}\right) \Gamma_{\nu}\right]_{a \sigma}{ }^{\prime}\left[\Gamma^{\nu} F\left(x-x^{\prime}\right)\right]_{\tau \beta}\right\} \varphi\left(\underset{\sigma}{x^{\prime}} \mid x_{\tau}^{\prime}\right)
\end{aligned}
$$

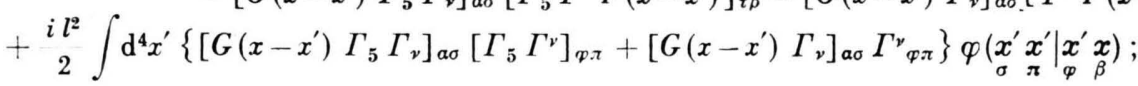


bei Iteration der zweiten Koordinate entsprechend

$$
\begin{aligned}
& \varphi\left(\left.\underset{\alpha}{x}\right|_{\beta} ^{x}\right)= \frac{i l^{2}}{2} \int \mathrm{d}^{4} x^{\prime}\left\{\left[F\left(x-x^{\prime}\right) \Gamma_{5} \Gamma_{\nu} G\left(x^{\prime}-x\right)\right]_{\alpha \beta}\left[\Gamma_{5} \Gamma^{\nu}\right]_{\tau \sigma}+\left[F\left(x-x^{\prime}\right) \Gamma_{\nu} G\left(x^{\prime}-x\right)\right]_{\alpha \beta} \Gamma_{\tau \sigma}^{\nu}\right. \\
&\left.-\left[F\left(x-x^{\prime}\right) \Gamma_{5} \Gamma_{\nu}\right]_{\alpha \sigma}\left[\Gamma_{5} \Gamma^{\nu} F\left(x-x^{\prime}\right)\right]_{\tau \beta}-\left[F\left(x-x^{\prime}\right) \Gamma_{\nu}\right]_{\alpha \sigma}\left[\Gamma^{\nu} F\left(x-x^{\prime}\right)\right]_{\tau \beta}\right\} \varphi\left(\left.\underset{\sigma}{x^{\prime}}\right|_{\tau} ^{x^{\prime}}\right) \\
&\left.+\frac{i l^{2}}{2} \int \mathrm{d}^{4} x^{\prime}\left\{\left[\Gamma_{5} \Gamma_{\nu} G\left(x^{\prime}-x\right)\right]_{\varrho \beta}\left[\Gamma_{5} \Gamma^{\nu}\right]_{\varphi \pi}+\left[\Gamma_{\nu} G\left(x^{\prime}-x\right)\right]_{\varrho \beta} \Gamma_{\varphi \pi}^{\nu}\right\} \varphi \underset{\alpha}{x} x_{\pi}^{\prime} \mid x_{\varphi} x_{\varrho}^{\prime}\right) .
\end{aligned}
$$

Gehören die 1-Teilchen-Matrixelemente $\varphi(x \mid x)$ zu einem Energie-Impuls $J^{\mu}$, so läßt sich die Ortsabhängigkeit abspalten

$$
\varphi\left(\left.\underset{\alpha}{x}\right|_{\beta} ^{x}\right)=\varphi_{a \beta} \exp \left(i J^{\mu} x_{\mu}\right) .
$$

Führt man wieder die in den früheren Arbeiten berechneten Funktionen von $X=\sqrt{\left|J^{2}\right| / \varkappa^{2}}$ ein

$$
\begin{aligned}
T_{\varepsilon \lambda}(X) & =J_{\varepsilon} J_{\lambda} D(X)+g_{\varepsilon \lambda} C(X) \\
& =-\frac{i l^{2} \varkappa^{4}}{(2 \pi)^{4}} \int \frac{\mathrm{d}^{4} p(J-p)_{\varepsilon} p_{\lambda}}{p^{2} N^{\prime}\left(p^{2}\right)}, \\
J_{\varepsilon} B(X) & =\frac{l^{2} \varkappa^{3}}{(2 \pi)^{4}} \int \frac{\mathrm{d}^{4} p(J-p)_{\varepsilon}}{N^{\prime}\left(p^{2}\right)}, \\
\text { wobei } & N^{\prime}\left(p^{2}\right)=(J-p)^{2}\left(p^{2}+\varkappa^{2}\right)\left(p^{2}\right)
\end{aligned}
$$

ist, das bei Integrationen entsprechend der FeynManschen Vorschrift behandelt werden soll, dann erhalten die Gln. $(107,108)$ bei Vernachlässigung der 4-Punktfunktionen und unter Berücksichtigung von (75) und (76) im Impulsraum die einfache Form:

$$
\begin{aligned}
& \varphi^{(1)}=\Gamma_{\varepsilon} M^{\prime}\left[\Gamma_{\lambda} T^{\varepsilon \lambda} Z_{p}+J^{\varepsilon} B Z_{\chi}\right], \\
& \varphi^{(2)}=\left[\Gamma_{\lambda} T^{\varepsilon \lambda} Z_{p}-J^{\varepsilon} B Z_{\varkappa}\right] M^{\prime} \Gamma_{\varepsilon}
\end{aligned}
$$

mit der Abkürzung

$$
\begin{aligned}
& M^{\prime}=\frac{1}{2}\left[\Gamma_{5} \Gamma_{\nu} \operatorname{Spur}\left(\Gamma_{5} \Gamma^{\nu} \varphi\right)\right. \\
& \left.\quad+\Gamma_{\nu} \operatorname{Spur}\left(\Gamma^{\nu} \varphi\right)-\Gamma_{5} \Gamma_{\nu} \varphi \Gamma_{5} \Gamma^{\nu}-\Gamma_{\nu} \varphi \Gamma^{\nu}\right] .
\end{aligned}
$$

Es ist nun zweckmäßig, $\varphi_{a \beta}$ auf die Dirac- und IsospinAlgebra aufzuspannen, und zwar sofort in einer Form, in der $\Gamma_{5}$ diagonal ist.

Beschränken wir uns zunächst auf Zustände, die zum Spin Null gehören, so treten nach „B“ IV B nur die Elemente auf

$$
\begin{aligned}
\varphi=\left[u_{11} R\left(\Gamma^{\mu} J_{\mu}^{0}\right)+u_{22}\right. & L\left(\Gamma^{\mu} J_{\mu}^{0}\right) \\
& \left.+u_{12} R+u_{21} L\right] M .
\end{aligned}
$$

Hierbei bedeutet $J_{\mu}^{0}=J_{\mu} / i \sqrt{\left|J^{2}\right|}$ einen Einheitsvektor in Richtung des Energie-Impulses $J^{\mu}$ und $u_{i k} \mathrm{Ko}$ effizienten, die nur von $X=\sqrt{\left|J^{2}\right| / x^{2}}=$ const abhängen. Die Abhängigkeit der Matrixelemente vom Isospin $\tau$ und den Vakuummatrizen $\varrho, \Sigma$ soll in $M$ enthalten sein. $R$ und $L$ sind die üblichen Projektionsoperatoren

$$
R=\frac{1}{2}\left(1+\Gamma_{5}\right), \quad L=\frac{1}{2}\left(1-\Gamma_{5}\right) .
$$

Nach einiger Rechnung ergibt sich für (113) und
(114) das folgende Gleichungssystem

$$
\begin{array}{r}
{\left[M+2 Q(X)(M+\operatorname{Sp} M) Z_{p}^{-}\right] u_{11}^{(1)}=0,} \\
{\left[M+2 Q(X)(M+\operatorname{Sp} M) Z_{p}^{+}\right] u_{22}^{(1)}=0,} \\
M u_{12}^{(1)}=2 i \varkappa X B(X)(M+\operatorname{Sp} M) Z_{\varkappa}^{+} u_{11}^{(1)}, \\
M u_{21}^{(1)}=2 i \varkappa X B(X)(M+\operatorname{Sp} M) Z_{\varkappa}^{-} u_{22}^{(1)} .
\end{array}
$$

und

$$
\begin{array}{r}
{\left[M+2 Q(X) Z_{p}^{-}(M+\operatorname{Sp} M)\right] u_{11}^{(2)}=0, \quad(122)} \\
{\left[M+2 Q(X) Z_{p}^{+}(M+\operatorname{Sp} M)\right] u_{22}^{(2)}=0, \quad(123)} \\
M u_{12}^{(2)}=-2 i \varkappa X B(X) Z_{\varkappa}^{+}(M+\operatorname{Sp} M) u_{22}^{(2)},(124) \\
M u_{21}^{(2)}=-2 i \varkappa X B(X) Z_{\varkappa}^{-}(M+\operatorname{Sp} M) u_{11}^{(2)} \cdot(125)
\end{array}
$$

Hierbei wurde die Abkürzung

$$
Q(X)=-J^{2} D(X)+2 C(X)
$$

und die Definitionen Gln. $(110,111)$ verwendet. Sp bedeutet nur eine Spur im Isospinraum ( $\tau$-Raum).

Da $\varphi$ von der Art der Näherung unabhängig, also $\varphi^{(1)}=\varphi^{(2)}$ oder $u_{i k}^{(1)}=u_{i k}^{(2)}$ sein soll, müssen die beiden Gleichungssysteme simultan erfüllt werden. Eliminiert man $u_{12}$ und $u_{21}$ aus den Gln. $(120,121,124,125)$, so ergeben sich zwei Bedingungsgleichungen für $u_{11}$ und $u_{22}$ :

$2 \varkappa X B(X)$

$\cdot\left[(M+\operatorname{Sp} M) Z_{\varkappa}^{+} u_{11}+Z_{\varkappa}^{+}(M+\operatorname{Sp} M) u_{22}\right]=0$,

$2 \varkappa X B(X)$

$\cdot\left[(M+\operatorname{Sp} M) Z_{x}{ }^{-} u_{22}+Z_{x}{ }^{-}(M+\operatorname{Sp} M) u_{11}\right]=0$.

Im allgemeinen werden sich die Gln. $(118,119 ; 122$, 123 ; 127, 128) nicht alle exakt erfüllen lassen, sie müssen also nach der im Abschnitt II a) geschilderten Methode behandelt werden.

Wir wollen das überbestimmte Gleichungssystem $(118-125)$ zuerst für den speziellen Fall des nichtentarteten Vakuums diskutieren, also $Z_{p}=Z_{\kappa}=1$ setzen. Dann ist auch $M=P_{\tau}{ }^{\mu} \tau_{\mu}$ und beschreibt nur Isosinglettzustände $\left(P_{\tau}^{0} \neq 0 ; P_{\tau}{ }^{k}=0\right)$ und Isotriplettzustände $\left(P_{\tau}{ }^{0}=0 ; P_{\tau}{ }^{k} \neq 0\right)$. Die Gln. $(118-125)$ reduzieren sich in diesem Fall auf die drei Beziehungen

$$
\begin{aligned}
{[M+2 Q(X)(M+\operatorname{Sp} M)]\left(u_{11}+u_{22}\right) } & =0, \\
{[M+2 Q(X)(M+\operatorname{Sp} M)]\left(u_{11}-u_{22}\right) } & =0, \\
2 \varkappa X B(X)(M+\operatorname{Sp} M)]\left(u_{11}+u_{22}\right) & =0 .
\end{aligned}
$$

Die ersten beiden Gleichungen sind die schon früher abgeleiteten identischen Eigenwertgleichungen für ein 
skalares bzw. pseudoskalares Boson. Neu gegenüber den alten Rechnungen ist hingegen Gl. (131), die eine Verträglichkeitsbedingung für die beiden Näherungen (107) und (108) ausdrückt. Sie läßt sich für Zustände mit endlicher Masse nur für die pseudoskalaren Lösungen $u_{11}+u_{22}=0$ und da sogar streng erfüllen. Die Lösungen mit positiver Parität werden also bei diesem Rechenverfahren - im Gegensatz zu früher - automatisch ausgeschaltet, da die Eigenparität des Nukleons in die Rechnung eingeht.

Da $X B(X)$ im Grenzfall verschwindender Bosonenmasse $\varkappa_{\mathrm{B}}=X \varkappa \rightarrow 0$ wie $X \ln X^{2}$ zu Null geht, könnte für Teilchen der Masse Null, wie schon in „B“ bemerkt wurde, u. U. die Forderung der Pseudoskalarität durchbrochen werden.

Bei Benutzung der Nebenbedingungen, wie wir die Gln. $(127,128)$ kurz nennen wollen, im Falle des entarteten Vakuums, bei welchen $Z_{p}$ und $Z_{\varkappa}$ von $\Gamma_{5}$ und $\tau$ abhängig werden, erscheint es äußerst unbefriedigend, daß nur $Z_{\varkappa}$ und nicht auch $Z_{p}$ eingeht, das doch zweifellos an der Parität etwas ändern muß. Umgekehrt gehen in die Hauptbedingungen, aus denen die Masseneigenwerte bezogen werden, die $Z_{\varkappa}$ gar nicht ein. Offensichtlich liegt dies an der Primitivität der Näherung. Es läßt sich leicht zeigen, daß schon in der nächsthöheren Näherung (Iteration einer 4-Punktfunktion) eine Verkopplung von $Z_{\mathrm{p}}$ und $Z_{\varkappa}$ in den Gleichungen auftritt. Leider werden die Rechnungen in dieser Näherung schon wesentlich komplizierter, so daß ihre Durchführung auf eine spätere Arbeit verschoben werden soll. Die Berechnungen über die seltsamen Bosonen (K-Mesonen) sind in dieser Arbeit deshalb noch reichlich unsicher.

Für Teilchen vom Spin 1 lassen sich ganz ähnliche Rechnungen durchführen, die hier nur nebenbei erwähnt werden sollen. Wir machen, entsprechend den Überlegungen in „B“ IV B den Ansatz

$$
\begin{gathered}
\varphi=\left[u_{11}{ }^{\prime} R\left(\Gamma_{\mu} P_{\sigma^{\mu}}{ }^{\prime}\right)+u_{22}{ }^{\prime} L\left(\Gamma_{\mu} P_{\sigma^{\mu}}{ }^{\mu}\right)\right. \\
\left.+u_{12}{ }^{\prime} R\left(\Gamma_{\mu \nu} J^{0 \mu} P_{\sigma}{ }^{\nu}\right)+u_{21}{ }^{\prime} L\left(\Gamma_{\mu \nu} J^{0 \mu} P_{\sigma}{ }^{\nu}\right)\right] M,
\end{gathered}
$$

wobei $P_{\sigma}$ den Polarisationsvektor bezeichnet, der wegen $P_{\sigma}{ }^{\mu} J_{\mu}=0$ durch die Spinrichtung des Teilchens im Ruhsystem festgelegt ist. Setzt man dies in Gl. (115) ein, so ergeben die Gln. $(113,114)$ nach einiger Rechnung

$$
\begin{array}{r}
{\left[M+2 Q^{\prime}(X)(M+\operatorname{Sp} M) Z_{p}^{-}\right] u_{11}^{\prime(1)}=0,} \\
{\left[M+2 Q^{\prime}(X)(M+\operatorname{Sp} M) Z_{p}^{+}\right] u_{22}^{\prime(1)}=0 ;} \\
M u_{12}^{\prime(1)}=2 i \varkappa X B(X)(M+\operatorname{Sp} M) Z_{\varkappa}^{+} u_{11}^{\prime(1)}, \\
M u_{21}^{\prime(1)}=2 i \varkappa X B(X)(M+\operatorname{Sp} M) Z_{\varkappa}^{-} u_{22}^{\prime(1)} ; \\
{\left[M+2 Q^{\prime}(X) Z_{p}^{-}(M+\operatorname{Sp} M)\right] u_{11}^{\prime(2)}=0,} \\
{\left[M+2 Q^{\prime}(X) Z_{p}^{+}(M+\operatorname{Sp} M)\right] u_{22}^{(2)}=0 ;} \\
M u_{12}^{\prime(2)}=2 i \varkappa X B(X) Z_{\varkappa}^{+}(M+\operatorname{Sp} M) u_{22}^{(2)}, \\
M u_{21}^{\prime(2)}=2 i \varkappa X B(X) Z_{\varkappa}^{-}(M+\operatorname{Sp} M) u_{11}^{\prime(2)},
\end{array}
$$

wobei die Abkürzung

$$
Q^{\prime}(X)=J^{2} D(X)+2 C(X)
$$

verwendet wurde.
Diese Gleichungen müssen wieder für $u_{i k}^{\prime(1)}=u_{i k}^{\prime(2)}$ simultan erfüllt werden.

Im Falle des nichtentarteten Vakuums lassen sich die Gln. $(133-140)$ auf die drei Beziehungen reduzieren

$$
\begin{gathered}
{\left[M+2 Q^{\prime}(X)(M+\operatorname{Sp} M)\right]\left(u_{11}{ }^{\prime}+u_{22}{ }^{\prime}\right)=0,} \\
{\left[M+2 Q^{\prime}(X)(M+\operatorname{Sp} M)\right]\left(u_{11}{ }^{\prime}-u_{22}{ }^{\prime}\right)=0,} \\
2 \varkappa X B(X)(M+\operatorname{Sp} M)\left(u_{11}{ }^{\prime}-u_{22}{ }^{\prime}\right)=0 .
\end{gathered}
$$

Die ersten beiden Gleichungen sind wieder die, schon in „B“ Gl. (106) angegebenen, identischen Eigenwertgleichungen für ein pseudoskalares bzw. skalares Boson vom Spin 1. Die Nebenbedingung (144) verbietet aber für endliche Massen $\left(X^{2} \neq 0\right)$ gerade wieder die Lösungen $\left(u_{11}^{\prime}-u_{22}{ }^{\prime}\right)$ mit positiver Parität $\left(P_{\sigma}\right.$ ist ein Pseudovektor!). Dies gilt nicht mehr für den Fall $X^{2}=0$, was wahrscheinlich für die Lichtquanten von Bedeutung sein wird.

Im übrigen wird es für Teilchen vom Spin 1 kaum zulässig sein, nur $\varphi\left(x \mid x^{\prime}\right)$-Funktionen am gleichen Punkt $x=x^{\prime}$ zu betrachten, d. h. nur s-Zustände einzubeziehen; aber diese Fragen sollen in der vorliegenden Arbeit nicht weiter verfolgt werden.

\section{c) Berechnung der Fermionen-Massen}

Für die folgende Abschätzung der Masseneigenwerte soll von einer Kontraktionsfunktion ausgegangen werden, in der, wie in der früheren Arbeit "A“, zur Vereinfachung nur ein Massenpol und die regularisierenden „Geisterdipol“-terme vorkommen. Die Massen aller Baryonen werden also als näherungsweise gleich angenommen, die Beiträge der kontinuierlichen Spektren durch die Regularisierungsterme ersetzt. Physikalisch soll der Massenpol etwa dem Schwerpunkt aller Baryonenmassen entsprechen. In Anlehnung an (8) und (27) kann die Kontraktionsfunktion daher (unter Weglassung der Indizes) in der Form geschrieben werden

$$
\begin{aligned}
& F\left(x-x^{\prime}\right)=(2 \pi)^{-4} \int \mathrm{d}^{4} p e^{i p\left(x-x^{\prime}\right)} \\
& \cdot\left\{\frac{p_{v} \Gamma^{v}\left(1+\alpha \Gamma^{5} \Sigma_{2}\right) \varkappa^{4}}{\left(p^{2}\right)^{2}\left(p^{2}+\varkappa^{2}\right)}-\frac{i \varkappa^{3} \beta\left[1+i \eta \Gamma^{5}(\varrho \tau) \Sigma_{3}\right]}{p^{2}\left(p^{2}+\varkappa^{2}\right)}\right\} .
\end{aligned}
$$

Für die Koeffizienten (78) gilt dann:

$Z_{p}=1+\alpha \Gamma_{5} \Sigma_{2} ; \quad Z_{\varkappa}=\beta\left(1+i \eta \Gamma_{5} \Sigma_{3}(\varrho \tau)\right)$.

Setzt man diese $Z$-Faktoren in die Gln. $(93-100)$ für die $\varphi$-Funktionen des vorigen Abschnitts ein und berücksichtigt dabei, daß bei der Multiplikation der Operatoren $\varrho, \Sigma$ stets das symmetrisierte Produkt (d. h. der Antikommutator) gemeint ist, so lassen sich diese Gleichungen - analog wie im Fall $Z_{p}=Z_{\varkappa}=1$ - wieder auf eine einzige Gleichung reduzieren. Man kann diese durch Multiplikation mit 
Ausdrücken wie

$$
\left(1-\alpha \Gamma_{5} \Sigma_{2}\right)^{-1} \text { und }\left(1+i \eta(\varrho \tau) \Gamma_{5} \Sigma_{3}\right)^{-1}
$$

auf die Normalform (36) bzw. (38) bringen. Man erhält so im Ruhsystem des betreffenden Teilchens

$$
\begin{aligned}
24\left(\frac{x l}{4 \pi}\right)^{4}\{ & Y\left(1-\alpha \Gamma_{5} \Sigma_{2}\right) \\
& \left.-\Gamma_{4}^{\prime}\left(1-i \eta(\varrho \tau) \Gamma_{5} \Sigma_{3}\right)\right\} \varphi=0,
\end{aligned}
$$

wobei

$$
Y=Y(X)=\frac{\beta\left(1+3 \eta^{2}\right)}{1-\alpha^{2}} \frac{X M(X)}{N(X)}
$$

gesetzt ist. Aus Gl. (101) folgt entsprechend unter Spezialisierung auf das Ruhsystem der betreffenden Masse:

$\left\{1+24\left(\frac{\varkappa l}{4 \pi}\right)^{4} L(X)\left(1+\alpha^{2}-2 \alpha \Gamma_{5} \Sigma_{2}\right)\right\} X \varphi=0$.

Die Variable $X$ in den nach II b) Gln. (80 bis 82 ) definierten Funktionen $L(X), M(X), N(X)$ bedeutet hierbei das Verhältnis der Masseneigenwerte zu der Schwerpunktmasse $\varkappa$. Die Gln. (147 bis 149) müssen nun zur Bestimmung der Baryonenmassen ausgenützt werden. Die Eigenwertgleichung für die Nukleonen soll hier dadurch gewonnen werden, daß man in den Gln. (147) und (149) zunächst über die Grundzustände mittelt, d. h. die Terme mit den Operatoren $\varrho$ und $\Sigma$ wegläßt. Denn die Nukleonen gehen aus einem symmetrischen Grundzustand hervor, eine Ankopplung von Spurionen findet nicht statt. $\mathrm{Da} ß$ trotzdem die Konstanten $\alpha$ und $\eta$ in den gemittelten Gleichungen vorkommen, hat seinen Grund darin, daß in den für die Nukleonen maßgebenden Graphen hier auch durch die Kontraktionsfunktion (145) - im Gegensatz zu den früheren Arbeiten "A“ und „B“ - seltsame Teilchenpaare als virtuelle Zwischenzustände zugelassen sind. Die neuen Gleichungen sollten daher die Massenverhältnisse zwischen Nukleonen und $\pi$-Mesonen etwas genauer darstellen als die früheren. Man erhält so für die $\mathrm{Nu}$ kleonen:

$$
\begin{gathered}
\left(Y-\Gamma_{4}\right) \varphi=0, \\
\left\{1+24\left(\frac{x l}{4 \pi}\right)^{4} L(X)\left(1+\alpha^{2}\right)\right\} X \varphi=0 .
\end{gathered}
$$

Da die erste Gleichung im Gleichungssystem (93 bis 101) wesentlich öfters vorkommt als die zweite, die nur aus (101) folgt, werden wir im folgenden

11 Selbstverständlich hat nur im Ruhsystem der Teilchen der Paritätsoperator diese einfache Form $\Gamma_{4}$ oder $\Gamma_{4} \Sigma_{1}$; im allgemeinen müssen noch die Raumkoordinaten gespiegelt werden, $d . h$. insbesondere $p_{k} \rightarrow-p_{k}$. die erste Gl. (147) bzw. (150) stets exakt zu lösen versuchen, uns aber bei der zweiten Gl. (149) bzw. (151) mit einer angenäherten Lösung begnügen. Dieses Verfahren wird gegenüber einer strengen Lösung des Minimalproblems (51), bei der man der ersten Gleichung ein entsprechend größeres Gewicht gibt als der zweiten, nur einen geringfügigen Fehler bewirken.

Für die Nukleonen folgt dann $\quad Y_{\mathrm{N}}=1$ und die Feststellung, daß die Parität ${ }^{11}$ der Nukleonen (entsprechend der ursprünglichen Definition) positiv ist:

$$
\Gamma_{4}=+1 \text {. }
$$

Für die Antinukleonen ist $Y_{\mathrm{N}}=-1$ und die Parität $\Gamma_{4}=-1$.

Für die Hyperonen läßt sich die Gl. (147) auch in der Form

$$
\left\{Y\left(1-\alpha \Gamma_{5} \Sigma_{2}\right)-\Gamma_{4}+\Gamma_{4} \Sigma_{1} \Gamma_{5} \Sigma_{2} \eta(\varrho \tau)\right\} \varphi=0
$$

anschreiben. Der Paritätsoperator ${ }^{11}$ heißt jetzt $\Gamma_{4} \Sigma_{1}$ und ist mit den Operatoren \{\} der Eigenwertgleichung (149) vertauschbar. Der Faktor $\Sigma_{1}$ im Paritätsoperator macht deutlich, daß die Parität gewissermaßen nur für ein Paar seltsamer Teilchen definiert werden kann, d. h., daß nur die Parität von Teilchen oder Teilchengruppen verglichen werden kann, die zum gleichen Wert der Seltsamkeit, der Baryonen- und Leptonenzahl, der Ladung usw. gehören ${ }^{12}$. Ferner kann für die Singletts ( $\Lambda$-Teilchen) $(\varrho \tau)=-3$, für die Tripletts ( $\Sigma$-Teilchen) $(\varrho \tau)=+1$ gesetzt werden. Man erhält so :

Für $\Gamma_{4} \Sigma_{1}=+1$ :

$Y_{\Lambda}=\frac{3 a \eta \pm \sqrt{1-\alpha^{2}+9 \eta^{2}}}{1-\alpha^{2}}, \quad Y_{\Sigma}=\frac{-\alpha \eta \pm \sqrt{1-\alpha^{2}+\eta^{2}}}{1-\alpha^{2}} ;$

für $\Gamma_{4} \Sigma_{1}=-1$ :

$Y_{\Lambda}=\frac{-3 \alpha \eta \pm \sqrt{1-\alpha^{2}+9 \eta^{2}}}{1-\alpha^{2}}, \quad Y_{\Sigma}=\frac{\alpha \eta \pm \sqrt{1-\alpha^{2}+\eta^{2}}}{1-\alpha^{2}}$.

Das negative Vorzeichen der Wurzel führt auch zu einem negativen Wert von $Y$, gehört also wegen (148) zu den Antiteilchen und braucht bei der Aufzählung der Teilchen nicht berücksichtigt zu werden. Für jeden Wert des Isospins gibt es daher zwei Masseneigenwerte, von denen wohl nur der niedrigere letztlich stabil sein kann. Diese tieferen Eigenwerte sollen daher mit den Hyperonen identifiziert werden.

12 Vgl. auch G. Lüders, Nuovo Cim. Rendiconti S. I. F. XI Corso, S. 9 [1961]. 
Die Parität $\Gamma_{4} \Sigma_{1}$ der zu den Massen gehörigen Teilchen hängt vom Vorzeichen des Produktes $a \eta$ ab. Bezeichnen wir dieses Vorzeichen mit $\operatorname{sgn}(\alpha \eta)$, so erhält man schließlich für die beiden Hyperonen, da die Funktion $X M(X) / N(X)$ in Gl. (148) mit $X$ anwächst:

A-Teilchen:

$$
\begin{aligned}
& Y_{A}=\frac{-3|\alpha \eta|+\sqrt{1-\alpha^{2}+9 \eta^{2}}}{1-\alpha^{2}} ; \quad(\varrho \tau)=-3, \\
& \text { Parität: } \quad \Gamma_{4} \Sigma_{1}=-\operatorname{sgn}(\alpha \eta) . \\
& \Sigma \text {-Teilchen: }
\end{aligned}
$$

$$
\begin{aligned}
& Y_{\Sigma}=\frac{-|\alpha \eta|+\sqrt{1-\alpha^{2}+\eta^{2}}}{1-\alpha^{2}} ; \quad(\varrho \tau)=+1, \\
& \text { Parität: } \quad \Gamma_{4} \Sigma_{1}=+\operatorname{sgn}(\alpha \eta) .
\end{aligned}
$$

Die Masse des $A$-Teilchens ist also kleiner als die des $\Sigma$-Teilchens, die relative Parität der beiden $\mathrm{Hy}$ peronen ist ungerade. Die Parität der Antiteilchen ist der Parität der Teilchen entgegengesetzt.

Bei der Bildung des Massenschwerpunkts wird man den Nukleonen, bei denen das Spurion entkoppelt ist, im ganzen das gleiche Gewicht geben müssen wie den Hyperonen zusammen. Man wird also setzen können:

$$
Y_{\mathrm{S}}=\frac{4 Y_{\mathrm{N}}+Y_{A}+3 Y_{\Sigma}}{8} .
$$

Da $M(X) / N(X)$ in Gl. (148) im Bereich der Baryonenmassen nicht wesentlich mit $X$ variiert, so wird diese Mittlungsvorschrift praktisch gleich einer Mittelung über die Massen der Baryonen selbst.

Für diesen Massenschwerpunkt sollte die Masse mit dem $\mathrm{Pol}$ in der Kontraktionsfunktion übereinstimmen, damit die Kontraktionsfunktion wenigstens in der hier beabsichtigten Näherung zum entstehenden Massenspektrum paßt; d. h.

$$
Y(1)=Y_{\mathrm{S}} \text {. }
$$

Diese Beziehung kann als eine Bestimmungsgleichung für die zunächst noch unbekannten Konstanten $\alpha$ und $\eta$ aufgefaßt werden.

Für die folgenden numerischen Rechnungen wollen wir

$$
|\eta| \ll|a|
$$

voraussetzen, was zunächst nur empirisch dadurch begründet werden kann, daß der Abstand zwischen $\Sigma$ - und $\Lambda$-Masse erheblich geringer ist als der zwischen $A$ - und Nukleonenmasse. Wir werden also in erster Näherung $\eta \approx 0$ setzen, d. h. die Aufspaltung in Singlett und Triplett vernachlässigen. Dann ist

$$
Y_{\mathrm{S}} \approx \frac{1}{2}\left(1+\frac{1}{\sqrt{1-a^{2}}}\right) \text {. }
$$

Wegen der schon in der früheren Arbeit "A“ erwähnten Werte: $L(1)=-0,6238 ; M(1)=1,9082$; $N(1)=2,3060$ folgt daraus

$$
\frac{\beta}{1-\alpha^{2}} \cdot 0,827 \approx \frac{1}{2}\left(1+\frac{1}{\sqrt{1-\alpha^{2}}}\right) .
$$

Der Wert von $\beta$ in der Kontraktionsfunktion (145) kann für jeden einzelnen Beitrag zu dieser Funktion leicht angegeben werden; für die Nukleonen allein wäre $\beta=1$, für die Hyperonen $\sqrt{1-\alpha^{2}}$. Im Mittel wird man, wegen des Regularisierungsfaktors $x^{4}$ in dieser Funktion, allerdings nicht ohne eine gewisse Willkür, ansetzen können:

$$
\bar{\beta}=\frac{1+\left(1-\alpha^{2}\right)^{-3 / 2}}{1+\left(1-\alpha^{2}\right)^{-2}}
$$

also $\quad 0,827 \frac{1+\left(1-\alpha^{2}\right)^{+3 / 2}}{1+\left(1-\alpha^{2}\right)^{2}}=\frac{1}{2}\left[1+\left(1-\alpha^{2}\right)^{1 / 2}\right]$,

daraus

$$
\alpha^{2} \approx 0,39 ; \quad \alpha \approx 0,625 ; \quad \beta \approx 0,84 ; \quad Y_{\mathrm{S}} \approx 1,14 .
$$

Die Berücksichtigung von $\eta$ würde den absoluten Wert von $\alpha$ verkleinern. Da der analytische Verlauf der Funktionen $L(X), M(X), N(X)$ bestenfalls eine recht grobe Näherung darstellen kann, muß auch der Zahlwert 0,827 als recht unsicher betrachtet werden. Die gewonnenen Werte für $\alpha$ und $\beta$ können nur als grobe Abschätzung gelten; das Vorzeichen von $\alpha$ kann ohne Einschränkung der Allgemeinheit als positiv angesetzt werden.

Bisher wurde die Eigenwertgleichung (149) noch nicht berücksichtigt. Wir können sie gleichzeitig für Nukleonen und Hyperonen mit einiger Genauigkeit befriedigen, wenn wir verlangen, da $\beta$ für den Schwerpunkt $X=1$ die Gleichung

$$
1+24\left(\frac{x l}{4 \pi}\right)^{4} L(1)\left(1+\alpha^{2}\right)=0
$$

gelten soll. Es lohnt hier kaum, das Minimalproblem (51) exakt unter Berücksichtigung des Verlaufs der Funktionen $L, M, N$ zu lösen. Man erhält bei dieser Näherung für den Schwerpunkt:

$$
\varkappa l=5,88,
$$

was zu vergleichen ist mit dem in der früheren Arbeit gewonnenen Wert $\varkappa l=6,39$.

Für die numerische Berechnung der Masseneigenwerte braucht man nun noch die Konstante $\eta$. Be- 
vor wir ihren Zahlwert diskutieren, sollen die Verhältnisse bei den Bosonen behandelt werden.

\section{d) Berechnung der Bosonen-Massen}

Die $\tau$ - bzw. $\varphi$-Funktionen der Bosonen waren in den Rechnungen von Abschnitt II b) als $u_{11}, u_{12}$, $u_{21}$ und $u_{22}$ bezeichnet worden; für sie wurden die Eigenwertgleichungen abgeleitet.

Für die folgenden Untersuchungen soll eine etwas andere Bezeichnung benützt werden, die die Ähnlichkeit mit den Fermionenrechnungen deutlicher hervortreten läßt. Die Eigenwertgleichungen (118 bis 125) lassen sich durch Elimination von $u_{12}$ und $u_{21}$ so umformen, daß nur Beziehungen zwischen $u_{11}$ und $u_{22}$ übrigbleiben [vgl. Gln. $\left.(127,128)\right] . u_{22}$ geht aus $u_{11}$ durch die Paritätsoperation hervor. Daher wollen wir einen Index $\gamma_{5}$ einführen, so daß

$$
\begin{array}{lll}
\text { für } & u_{11} & \gamma_{5}=+1, \\
\text { für } & u_{22} & \gamma_{5}=-1 \text { ist. }
\end{array}
$$

Der Paritätsoperator lautet dann $\gamma_{4}$, soweit er sich auf die Feldoperatoren bezieht, oder allgemeiner $\gamma_{4} \Sigma_{1}$. Der Operator $\gamma_{4}$ entspricht also etwa einem Produkt $\Gamma_{4} \cdot \Gamma_{4}$, das auf die beiden Fermionenanteile der Bosonen wirkt. Die hier eingeführten $\gamma_{4}$ und $\gamma_{5}$ haben natürlich nichts mit den $\gamma_{4}$ und $\gamma_{5}$ der Ausgangsgleichung (1) zu tun.

Ferner beziehen sich gewisse Anteile von $M$ auf einen Iso-Triplettzustand $\left(M=P_{\sigma}{ }^{k} \tau_{k}\right)$, andere auf einen Iso-Singlettzustand $\left(M=P_{\sigma}{ }^{0} \tau_{0}=P_{\sigma}{ }^{0}\right)$ des durch die beiden Feldoperatoren dargestellten Gebildes. Es ist zweckmäßig, diese beiden Anteile durch einen weiteren Index zu unterscheiden, den wir $\sigma_{3}$ nennen wollen (der aber natürlich nichts mit dem Dirac-Spin zu tun haben soll). Wir setzen also fest:

$$
\begin{array}{ll}
\text { Singlettanteil von } M: & \sigma_{3}=+1, \\
\text { Triplettanteil von } M: & \sigma_{3}=-1 .
\end{array}
$$

Die erste Gruppe der Eigenwertgleichungen $[(118,119)$ und $(122,123)]$ für die Bosonen erhält unter Benützung dieser Bezeichnungen und Einsetzung der Kontraktionsfunktion (145) die Form

$$
\left[1+2 Q\left(1-\alpha \gamma_{5} \Sigma_{2}\right)\left(2+\sigma_{3}\right)\right] u=0,
$$

wobei $Q(X)$ die in II b) Gln. $(126,110)$ definierte und in der früheren Arbeit "A “ berechnete Funktion des Verhältnisses $X$ der Bosonenmasse zur Schwerpunktmasse der Fermionen ist. In dieser Gleichung kommt die Ankopplung des Spurion-Isospins an den Isospin des $\pi$-Mesons noch nicht zum Ausdruck.
Eine zweite Eigenwertgleichung erhält man aus den Beziehungen $(127,128)$. Die dort nach Einsetzung der Z-Faktoren (146) auftretenden Operatoren $(\varrho \tau)$, die einmal von links, ein anderes Mal von rechts auf die Isospinindizes in $M$ angewandt werden müssen, drücken sich in den Indizes $\sigma_{3}$ verschieden aus, je nachdem, ob es sich bei dem aus Spurion und $\pi$-Meson bestehenden System um ein Quartett- oder Dublettsystem handelt. Insbesondere stammt das Quartettsystem vom Triplett-Spurionensystem her, und es ist dann

$$
M=\vec{Q}_{a} \cdot\left[\delta_{\alpha \beta} \vec{\tau}-\frac{i}{2}\left(\vec{\varrho}_{\alpha \beta} \times \vec{\tau}\right)\right] v_{\beta} ;
$$

Dublettsysteme resultieren dagegen sowohl von Triplett-Spurionensystemen mit

$$
M=D_{a} \frac{1}{\sqrt{3}}\left(\vec{\varrho}_{\alpha \beta} \cdot \vec{\tau}\right) v_{\beta},
$$

als auch von Singlett-Spurionensystemen mit

$$
M=D_{\alpha} v_{\alpha},
$$

wobei $\vec{Q}_{a}$ und $D_{\alpha}$ die Zustände des Quartetts bzw. Dubletts charakterisieren. Man verifiziert dann leicht für das

Quartettsystem:

$\overrightarrow{(\varrho \tau)}=1 ; \quad \overleftarrow{(\varrho \tau)}=-1$,

Dublettsystem:

$\widehat{(\varrho \tau)}=-1+\sigma_{3}+\sqrt{3} \sigma_{1} ; \quad \overleftarrow{(\varrho \tau)}=1-\sigma_{3}+\sqrt{3} \sigma_{1}$.

[Die Pfeile in (172) geben an, in welcher Richtung der Operator wirkt.]

Benützt man noch die Abkürzung

$$
w=\left(2+\sigma_{3}\right) u,
$$

so erhält die zweite Eigenwertgleichung die Form: Quartettsystem:

$$
2 \varkappa X B \beta\left(1+\gamma_{4}\right)\left(1-i \eta \gamma_{5} \Sigma_{3}\right) w=0,
$$

Dublettsystem:

$$
\begin{array}{r}
2 \varkappa X B \beta\left\{\left(1+\gamma_{4}\right)\left[1+i \eta \Sigma_{3} \gamma_{5}\left(1-\sigma_{3}\right)\right] \quad\right. \\
\left.+\left(1-\gamma_{4}\right) i \eta \sqrt{3} \Sigma_{3} \gamma_{5} \sigma_{1}\right\} w=0 .
\end{array}
$$

Diese zweite Eigenwertgleichung enthält, worauf schon hingewiesen wurde, nur die Konstante $\eta$, nicht aber $\alpha$; sie erweist sich dadurch als eine allzu grobe Näherung, in der eine numerische Bestimmung der Konstante $\eta$ noch nicht durchgeführt werden kann.

Um die Masse der $\pi$-Mesonen zu berechnen, soll in den Gleichungen über die Grundzustände gemit- 
telt, also alle Glieder mit den Operatoren $\varrho, \Sigma$ weggelassen werden. Die beiden Eigenwertgleichungen können dann gleichzeitig streng erfüllt werden. Die Gl. (131) sagt nur aus, daß die $\pi$-Mesonen ungerade Parität $\left(\gamma_{4}=-1\right)$ besitzen. Für das Verhältnis der Bosonenmasse $\varkappa_{\mathrm{B}}$ zur Masse des Fermionenschwerpunkts $\varkappa$ folgt bei den $\pi$-Mesonen:

$$
\begin{array}{ll}
\text { Iso-Triplett: } & Q=-1 / 2 ; \quad \varkappa_{\mathrm{B}} / \varkappa=0,170 ; \\
\text { Iso-Singlett: } & Q=-1 / 6 ; \quad \varkappa_{\mathrm{B}} / \varkappa=0,665 .
\end{array}
$$

Würde das Isosinglett-Teilchen in einer höheren Näherung noch etwas schwerer werden, so dürfte es gegenüber einem Zerfall in vier Triplett-Teilchen instabil werden. Die Änderung der Zahlwerte gegenüber denen der früheren Arbeit "A“ ist durch den niedrigeren Wert von $\varkappa l(5,88$ gegenüber 6,39$)$, d. h. durch die Berücksichtigung der seltsamen Teilchen in den Zwischenzuständen bedingt.

Bei der Berechnung der Bosonen mit angekoppeltem Spurion wollen wir zunächst von der Eigenwertgleichung (168) ausgehen, da die andere Gleichung durch den Faktor $2 \varkappa \beta X B$ wenigstens bei kleinen Bosonenmassen ein relativ kleines Gewicht hat. (Man kann durch Ausrechnen der Integrale zeigen, daß $|\varkappa B(X)|=|Q(X)|$ ist.) Es ergeben sich dann vier Eigenwerte für die vier Wertekombinationen $\sigma_{3}= \pm 1 ; \quad \Sigma_{2} \gamma_{5}= \pm 1$.

$\boldsymbol{\sigma}_{\mathbf{3}}=+\mathbf{1}$ :

$\Sigma_{2} \gamma_{5}=-1, \quad Q=-\frac{1}{c(1+c)}, \quad \varkappa_{\mathrm{B}}=0,804$,

$\Sigma_{2} \gamma_{5}=+1, \quad Q=-\frac{1}{6(1-\alpha)}, \quad \varkappa_{\mathrm{B}}=0,216$,

$\boldsymbol{\sigma}_{3}=-1$ :

$\Sigma_{2} \gamma_{5}=-1, \quad Q=-\frac{1}{2(1+\alpha)}, \quad \varkappa_{\varkappa_{\mathrm{B}}}=0,394$,

$\Sigma_{2} \gamma_{5}=+1, \quad Q=-\frac{1}{2(1-\alpha)}, \quad \varkappa_{\mathrm{B}}=0,00484$.

Der erste Eigenwert gehört zu einem Isodublett. Er liegt viel höher als andere Zustände gleicher Symmetrie, wird also nicht ein stabiles Teilchen darstellen können. Der zweite Eigenwert gehört zu einem anderen Isodublett, der dritte kann Isoquartett und Isodublett sein. Diese Zustände liegen relativ nahe beisammen und könnten durch eine kleine Änderung von $\alpha(\alpha=0,5$ statt 0,625$)$ ganz zusammenrücken. Der vierte Eigenwert schließlich liegt im Gebiet der Elektronenmassen; er wird aber, wenn der Gewichtsfaktor der zweiten Eigenwertgleichung (174) und (175) nicht zu klein ist, nach II a) kaum mehr als echter Eigenwert angesehen werden können. Wir wollen diesen unsicheren Eigenwert also nicht weiter diskutieren.

Berücksichtigt man die zweite Eigenwertgleichung (174) und (175) im Sinne der Minimalforderung (51), so werden sich alle Eigenwerte etwas verschieben, außerdem wird diese Gleichung wie bei den Fermionen einen Wert der Parität vor dem anderen auszeichnen, indem der Fehler der TаммDancoff-Gleichungen für die eine Parität kleiner wird als für die entgegengesetzte. Wir wollen, mit den Vorbehalten von II a), jeweils diesen Eigenwert und die zugehörige Parität als die „richtigen“ ansehen.

Die beiden mittleren Eigenwerte (die durch Berücksichtigung der zweiten Eigenwertgleichung noch etwas verschoben und vom Wert von $\eta$ abhängig werden können), liefern neben dem Quartett-Term zwei Dubletts, die wir mit den K-Mesonen identifizieren wollen.

Bei einer PG-Transformation der Feldoperatoren gehen die Zustandsvektoren des aus dem Iso-Triplett stammenden Dubletts in sich über, die aus dem Isosinglett stammenden wechseln ihr Vorzeichen. Wenn die Eigenwertgleichung für die K-Mesonen neben den bisher betrachteten Symmetrien auch noch die weitere hat, $\mathrm{da} ß$ sie bei einer Permutation aller drei Isospins (zwei von den Feldoperatoren, einer vom Spurion) invariant bleibt, so fallen die beiden $\mathrm{Du}$ bletts zusammen. Es wird also unter Umständen möglich sein, die Kopplungskonstante $\eta$, die für die Ankopplung des Spurion-Isospins maßgebend ist, so zu wählen, daß diese weitere Invarianz besteht und die Dubletts zusammenfallen. Erst dann entstehen vier Zustände gleicher Masse, die genau die Symmetrieeigenschaften der beobachteten K-Mesonen aufweisen. Diese weitere Invarianz wird also zwar durch die bei den Kontraktionsfunktionen angenommenen Symmetrien nicht erzwungen, sie wird von ihnen aber zugelassen und kann bei der angenommenen Symmetrie der vier Grundzustände $\left.\Omega_{a}\right\rangle$ als naturgemäß gelten. Die volle Bedeutung der weiteren Symmetrieforderung wird wohl erst im $\mathrm{Zu}$ sammenhang mit der Elektrodynamik (Begriff der elektrischen Ladung) klargestellt werden können, doch soll hierauf an dieser Stelle nicht eingegangen werden (vgl. II e).

Wir führen die Rechnung zunächst für den bei $\varkappa_{\mathrm{B}} / \varkappa=0,394$ liegenden Quartett-Term durch. Für diesen Term kann $\sigma_{3}=-1$ und $\Sigma_{2} \gamma_{5}=-1$ gesetzt, 
die Wechselwirkung mit anderen Termen vernachlässigt und die quadrierte zweite Eigenwertgleichung durch ihren Erwartungswert für Funktionen des Typus $\sigma_{3}=-1, \Sigma_{2} \gamma_{5}=-1$ ersetzt werden. Wenn die Gl. (168), da sie zweimal auftritt, mit doppeltem Gewicht eingesetzt wird, lautet die Minimalforderung (51) :

$$
\begin{aligned}
2[1+2(1+\alpha) Q]^{2}+(2 \varkappa X B \beta)^{2} \\
\cdot 2\left[1+\eta\left(\gamma_{4} \Sigma_{1}\right)\right]^{2}=\operatorname{Min} .
\end{aligned}
$$

Man erkennt, daß der negative Wert der Parität: $\gamma_{4} \Sigma_{1}=-1$ zum kleineren Wert des Fehlers führt, wenn $\eta>0$ gewählt wird. Bei allgemeinerer Wahl des Vorzeichens von $\alpha$ und $\eta$ würde sich, ähnlich wie bei den Fermionen für das

$$
\text { Quartett: Parität } \gamma_{4} \Sigma_{1}=-\operatorname{sgn}(\alpha \eta)
$$

ergeben. Die geringfügige Verschiebung des Eigenwerts durch den zweiten Term in (178) soll hier nicht berechnet werden.

Für die beiden Dubletts, die zunächst bei $\varkappa_{\mathrm{B}} / \varkappa=0,216$ und 0,394 liegen, kann $\Sigma_{2} \gamma_{5} \sigma_{3}=+1$ gesetzt werden. Vernachlässigt man die Wechselwirkung mit anderen Termen, so läßt sich die quadrierte zweite Eigenwertgleichung (175) durch ihren Erwartungswert für Funktionen, bei denen $\Sigma_{2} \gamma_{5} \sigma_{3}=+1$ ist, ersetzen. Als Minimalforderung ergibt sich dann:

$2\left[\frac{2-\sigma_{3}}{3}+2 Q\left(1-\alpha \sigma_{3}\right)\right]^{2}+(2 \varkappa X B \beta)^{2}$

$\cdot 2\left[1-2 \eta\left(\gamma_{4} \Sigma_{1}\right)\left(1-\sigma_{3}\right)+\eta^{2}\left(5-2 \sigma_{3}\right)\right]=$ Min .

Für die Parität folgt hieraus, bei Wahl eines positiven $\eta:\left(\gamma_{4} \Sigma_{1}\right)=+1$, also allgemeiner für das

K-Meson: Parität $\gamma_{4} \Sigma_{1}=+\operatorname{sgn}(\alpha \eta)$.

Versucht man nun weiter, der oben angestellten Überlegung entsprechend, den Wert von $\eta$ so $\mathrm{zu}$ wählen, daß der durch die Minimalforderung bestimmte Eigenwert $X$ von $\sigma_{3}$ unabhängig wird, d. h. daß die beiden Dubletts zusammenfallen, so erweist sich dies wegen des Verhaltens von $2 \varkappa X B \beta$ als Funktion von $Q$ (oder $X$ ) zunächst als unmöglich.
Allerdings würden sich bei Veränderungen des „Gewichtsverhältnisses" der beiden Gleichungen solche Lösungen ergeben; aber eine Bestimmung von $\eta$, die stark vom Gewichtsverhältnis abhängt, kann nicht zu zuverlässigen Werten führen. Man müßte an dieser Stelle also zu höheren TAMm-DancofFNäherungen übergehen und könnte hoffen, dann aus der Forderung des Zusammenfallens der beiden Dubletts den Wert von $\eta$ zu berechnen. Da dazu sehr komplizierte Rechnungen durchgeführt werden müßten, soll auf eine theoretische Berechnung von $\eta$ im Rahmen der vorliegenden Arbeit verzichtet werden. Wir wollen $\eta$ so wählen, daß die empirischen Masseneigenwerte der Hyperonen möglichst gut dargestellt werden. Als der beste Wert ergibt sich dabei etwa

$$
\eta \approx 0,03 \text {. }
$$

Die Masse des K-Mesons im Vergleich zum QuartettTerm läßt sich in der Weise abschätzen, daß man nur die von $\sigma_{3}$ unabhängigen Anteile des ersten Ausdrucks von (180) zum Minimum macht. Man erhält so für das K-Meson :

$$
\begin{aligned}
& Q=-\frac{2+\alpha}{6\left(1+\alpha^{2}\right)}=-0,314 ; \\
& X=\frac{\varkappa_{\mathrm{B}}}{\varkappa}=0,384 .
\end{aligned}
$$

Die kleine Korrektur durch den zweiten Term in (180) ist wie beim Quartett unberücksichtigt geblieben. Die das K-Meson darstellenden Dublettzustände liegen also etwas, aber nur wenig tiefer als die Isoquartettzustände. Die letzteren wären nach den hier vorliegenden Rechnungen bestenfalls gegenüber der Aussendung von $\gamma$-Quanten instabil.

Wenn man die Werte $\alpha=0,625$ und $\eta=0,03$ in die Formeln (155-157) einsetzt, so erhält man schließlich die in Tab. 1 zusammengefaßten Massen-

\begin{tabular}{|c|c|c|c|c|c|c|c|c|c|c|c|c|}
\hline Teilchen & & $N \bar{N}$ & $\Lambda \bar{\Lambda}$ & $\Sigma \bar{\Sigma}$ & $\Lambda^{\mathrm{i}} \overline{\Lambda^{\mathrm{i}}}$ & $\sum \mathrm{i} \overline{\Sigma i}$ & $\pi_{\mathrm{Tr}}$ & $\pi_{\mathrm{s}}^{\mathrm{i}}$ & $\mathrm{K} \overline{\mathrm{K}}$ & $\mathrm{K}_{\mathrm{Qu}}^{i}$ & $\mathbf{K}_{\text {Dubl }}^{i}$ & $?$ \\
\hline Masse & $\begin{array}{l}\text { theor. } \\
\text { beob. }\end{array}$ & $\begin{array}{l}1 \\
1\end{array}$ & $\begin{array}{l}1,195 \\
1,19\end{array}$ & $\begin{array}{l}1,250 \\
1,265\end{array}$ & $1,36.5$ & 1,312 & $\begin{array}{l}0,194 \\
0,1485\end{array}$ & 0,76 & $\begin{array}{l}0,437 \\
0,532\end{array}$ & 0,448 & 0,915 & 0,0055 \\
\hline $\begin{array}{l}\text { Relative } \\
\text { Parität }\end{array}$ & $\begin{array}{l}\text { theor. } \\
\text { beob. }\end{array}$ & $\begin{array}{l}+- \\
+\quad-\end{array}$ & -+ & +- & +- & -+ & - & - & + & - & & \\
\hline
\end{tabular}
eigenwerte.

Die Masse des Nukleons ist dabei als Maßstab $=1$ gesetzt. Instabile Zustände sind durch die in der Symmetrie entsprechenden Teilchensymbole und mit dem Index i bezeichnet. Das gewöhnliche $\pi$ -

Tab. 1. Masseneigenwerte. 
Meson wird als Isotriplett mit $\pi_{\mathrm{Tr}}$, das (instabile) Isosinglett mit $\pi_{\mathrm{s}}{ }^{\mathrm{i}}$ angeführt. Die letzte Spalte enthält den scheinbaren Bosonen-Eigenwert in der Nähe der Elektronenmasse, der wegen der Nebenbedingung wahrscheinlich bei genauerer Rechnung wegfiele.

Als relative Parität wird der Wert von $\Gamma_{4}, \gamma_{4}$ oder $\Gamma_{4} \Sigma_{1}, \gamma_{4} \Sigma_{1}$, unter Weglassung des für alle seltsamen Teilchen gemeinsamen Faktors $\operatorname{sgn}(\alpha \eta)$ angegeben ${ }^{12}$. Es ist dabei hervorzuheben, daß auch empirisch nur die Parität von Teilchen oder Teilchengruppen verglichen werden kann, die zum gleichen Wert von Ladung, Baryonen- und Leptonenzahl und $z$-Komponente des Isospins gehören; der Faktor $\operatorname{sgn}(\alpha \eta)$ fällt also heraus.

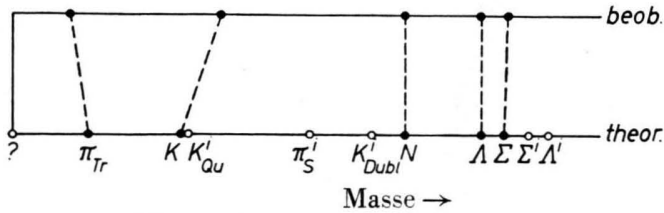

Abb. 1. Masseneigenwerte der seltsamen Teilchen.

In Abb. 1 sind die numerischen Verhältnisse noch einmal anschaulich dargestellt. Die Massen der stabilen oder nahezu stabilen Teilchen werden von der Theorie qualitativ richtig wiedergegeben. Für die Hyperonen ist die Übereinstimmung mit den empirischen Massen sogar besser als es in der ersten Näherung der TAMm-Dancoff-Methode erwartet werden sollte; dies rührt davon her, daß die Konstante $\eta$ nicht aus der Theorie, sondern empirisch bestimmt wurde. Dadurch wäre es möglich, eine der Hyperonenmassen sogar exakt mit dem empirischen Wert übereinstimmen zu lassen. Die relative Lage der verschiedenen Masseneigenwerte würde sich aber auch bei einer erheblichen Variation der Konstanten $\alpha$ und $\eta$ nur wenig ändern.

Uber die Parität der seltsamen Teilchen ist bisher experimentell nur wenig bekannt. BLocK ${ }^{13}$ und Mitarbeiter haben die relative Parität von $\mathrm{K}^{-}+\mathrm{P}$ und $\Lambda$ mit Hilfe des Prozesses $\mathrm{K}^{-}+\mathrm{He}^{4} \rightarrow{ }_{\Lambda} \mathrm{He}^{4}+\pi^{-}$ untersucht und gefunden, da $\beta$ diese relative Parität wahrscheinlich negativ ist, wie die vorliegende Theorie es auch fordert. Doch sind die experimentellen Ergebnisse noch nicht ganz eindeutig. Verschiedene andere Experimente zur Bestimmung der relativen

13 M. M. B Lock u. Mitarbeiter, Bericht von der Rochester-Konferenz 1960, S. 419.

14 Ph. Meyer, J. Prentki u. Y. Yamaguchi, Phys. Rev. Lett. 5 , 442 [1960]. - M. Muraskin, Phys. Rev. 119, 818 [1960]. - J. Sucher u. G. A. Snow, Nuovo Cim. 18, 195 [1960].
Paritäten $\Sigma-\Lambda^{14}, \mathrm{~K}-\Lambda^{15}, \mathrm{~K}-\Sigma^{16}$ sind vorgeschlagen, aber noch nicht ausgeführt worden. Die theoretischen Voraussagen der Tab. 1 über die Parität können also erst später experimentell geprüft werden.

\section{e) Die Symmetrieeigenschaften der K-Mesonen und der Begriff „Seltsamkeit“ (strangeness)}

Die in $I$ b) und c) geforderte GP-Symmetrie der vier Grundzustände hatte nicht ausgereicht, um das Zusammenfallen der beiden Dubletts im K-Meson zu erzwingen. Vielmehr mußte noch eine besondere Forderung gestellt werden, die z. B. als Permutationssymmetrie der Isospins ausgedrückt werden kann. Um zu zeigen, daß diese Symmetrie und damit der Begriff „strangeness“ eng mit der Definition der elektrischen Ladung verknüpft ist, sollen die Symmetrieverhältnisse zunächst an den empirischen K-Mesonen studiert werden. Bei Anwendung der Operation GP auf die K-Mesonen wird man etwa zu folgender Festsetzung kommen können:

$$
\begin{array}{ll}
\left.\mathrm{GP} \cdot \mathrm{K}^{+}\right\rangle=\overline{\left.\mathrm{K}^{0}\right\rangle,} & \left.\left.\mathrm{GP} \cdot \mathrm{K}^{-}\right\rangle=\mathrm{K}^{\mathbf{0}}\right\rangle, \\
\left.\left.\mathrm{GP} \cdot \overline{\mathrm{K}}^{0}\right\rangle=\mathrm{K}^{+}\right\rangle, & \left.\left.\mathrm{GP} \cdot \mathrm{K}^{\mathbf{0}}\right\rangle=\mathrm{K}^{-}\right\rangle .
\end{array}
$$

Daraus folgt dann:

$$
\begin{aligned}
& \left.\left.\left.\left.\mathrm{GP} \cdot\left(\mathrm{K}^{+}\right\rangle+\overline{\mathrm{K}}^{0}\right\rangle\right)=\mathrm{K}^{+}\right\rangle+\overline{\mathrm{K}}_{\mathbf{0}}\right\rangle, \\
& \left.\left.\left.\left.\mathrm{GP} \cdot\left(\mathrm{K}^{+}\right\rangle-\overline{\mathrm{K}}^{0}\right\rangle\right)=-\left(\mathrm{K}^{+}\right\rangle-\overline{\mathrm{K}}_{\mathbf{0}}\right\rangle\right) .
\end{aligned}
$$

Beim Vergleich mit den Rechnungen in Abs. II d wird man also folgende Zuordnung annehmen müssen:

\begin{tabular}{|c|c|c|}
\hline Isospin & \multicolumn{1}{c|}{} & $-\frac{1}{2}$ \\
\hline $\begin{array}{l}\text { 1. Dublett } \\
\text { (aus Singlett) }\end{array}$ & $\left.\left.\frac{1}{\sqrt{2}}\left(\mathrm{~K}^{+}\right\rangle-\overline{\mathrm{K}}^{0}\right\rangle\right)$ & $\left.\left.\frac{1}{\sqrt{2}}\left(\mathrm{~K}^{-}\right\rangle-\mathrm{K}^{0}\right\rangle\right)$ \\
$\begin{array}{l}\text { 2. Dublett } \\
\text { (aus Triplett) }\end{array}$ & $\left.\left.\frac{1}{\sqrt{2}}\left(\mathrm{~K}^{+}\right\rangle+\overline{\mathrm{K}}^{0}\right\rangle\right)$ & $\left.\left.\frac{1}{\sqrt{2}}\left(\mathrm{~K}^{-}\right\rangle+\mathrm{K}^{0}\right\rangle\right)$ \\
\hline
\end{tabular}

Wenn nun die beiden Dubletts energetisch verschieden wären, d. h. wenn die erste Zeile zu einem anderen Masseneigenwert als die zweite gehörte, so wäre der Zustand $\left.\mathrm{K}^{+}\right\rangle$kein stationärer Zustand mehr, da er durch Zusammenfügen zweier Zustände

15 L. Okun u. I. Ia. Pomeranchuk, J. Exp. Theor. Phys., USSR 34, 997 [1958].

16 W. M. Frank, I. Goldberg u. R. M. Rockmore, Phys. Rev. 117, 1402 [1960]. 
verschiedener Energie entstände. Der Zustand $\mathrm{K}^{+}$) müßte sich nach Ablauf der halben durch die Massendifferenz gegebenen Periode in den Zustand $\left.\overline{\mathrm{K}}^{\mathbf{0}}\right\rangle$ verwandeln. Die elektrische Ladung wäre dann nicht mehr erhalten. Umgekehrt folgt also aus der Erhaltung der Ladung, daß die beiden Dubletts notwendig zusammenfallen müssen. (Von Energiebeträgen elektromagnetischer Größenordnung wird dabei abgesehen.)

Allerdings läßt sich diese Schlußweise im Rahmen der vorliegenden Theorie einstweilen nicht verwenden, da ja noch nicht gezeigt ist, daß die Gl. (1) wirklich auch zu einer Elektrodynamik führt. In einer früheren Arbeit ${ }^{17}$ wurde für eine Theorie etwas anderer Struktur nachgewiesen, daß dort Operatoren vom Typ

$$
\bar{\psi}\left(\Gamma_{\mu} \Gamma_{v}-\Gamma_{v} \Gamma_{\mu}\right) \psi
$$

die Rolle von Feldoperatoren der Lichtquanten spielen und daß in dieser Weise eine Elektrodynamik entsteht. Die entsprechende Untersuchung ist in der vorliegenden Theorie aber noch nicht durchgeführt worden. Andererseits ist es plausibel,

17 R. Ascoli u. W. Heisenberg, Z. Naturforschg. 12 a, 177 [1957]. daß das Verhalten von Vakuumerwartungswerten von der Art (17) eng mit dem Auftreten von Kräften langer Reichweite verknüpft ist.

Daß man den Satz von der Erhaltung der „Seltsamkeit“ (strangeness) auf den Satz von der Erhaltung der Ladung zurückführen kann, wenn man die übrigen Erhaltungssätze (Isospin, Baryonen- und Leptonenzahl) schon besitzt, ist seit langem bekannt. Wir müssen also auch in der vorliegenden Untersuchung die Definition der Quantenzahl „Seltsamkeit“ auf einen späteren Zeitpunkt verschieben, bis eine Ableitung der Elektrodynamik durchgeführt ist. In diesem Zusammenhang sollte dann auch eine genauere Bestimmung der beiden Konstanten $\alpha$ und $\eta$ möglich werden. Die erwähnte frühere Untersuchung macht es wahrscheinlich, daß die numerische Bestimmung von $e^{2} / \hbar c$ eng mit der von $\alpha$ und $\eta$ zusammenhängt.

Wenn man im Rahmen der vorliegenden Untersuchung Teilchen höherer Seltsamkeit, d. h. $\Xi$-Teilchen darstellen wollte, müßte man entweder im Sinne der Gl. (17) Erwartungswerte für Produkte aus sechs Feldoperatoren studieren, oder eine höhere Entartung des Grundzustandes explizite in Betracht ziehen. Doch sollen diese Rechnungen hier nicht mehr durchgeführt werden. 